\title{
Non-homogeneous boundary value problems for linear dispersive equations
}

\author{
Corentin Audiard \\ Institut Camille Jordan, Université Claude Bernard Lyon
}

\begin{abstract}
While the non-homogeneous boundary value problem for elliptic, hyperbolic and parabolic equations is relatively well understood, there are still few results for general dispersive equations. We define here a convenient class of equations comprising the Schrödinger equation, the Airy equation and linear 'Boussinesq type' systems, which is in some sense a generalization of strictly hyperbolic equations, and for which we define a generalized Kreiss-Lopatinskii condition. From the construction of generalized Kreiss symmetrizers (adapted from hyperbolic theory) we deduce a priori estimates and well posedness for the pure boundary value problems (BVP) on a half-space associated to this class of equations. The initial boundary value problem (IBVP) is investigated too for the special case of the Schrödinger equation, and possible generalizations of the proof for other problems are indicated. Keywords: Boundary value problems, dispersive equations, Kreiss symmetrizers.
\end{abstract}

\section{Introduction}

The analysis of boundary value problems for dispersive equations like the Schrödinger equation or the (non-linear) wave equation has received a lot of attention during the last ten years. In the case of homogeneous Dirichlet or Neumann boundary conditions, sharp results (Kato-smoothing of the trace and Strichartz estimates) have been obtained under various geometric assumptions, see Burq-Gerard-Tzvetkov [5], Burq [4], Ivanovici [13], Planchon-Vega [20]... For the non-homogeneous boundary conditions, the well-posedness of the Korteweg-de Vries equation posed on a quarter plane or a strip was obtained independently by Bona-Sun-Zhang [2,3] and Faminskii-Larkine [8, 9]. These results were based on a priori estimates following from explicit computations of the solutions of linearized equations. In the same spirit, Fokas [10] proved the well-posedness of a large class of one-dimensional linear scalar dispersive equations.

In several dimensions of space, these techniques do not apply, notably because of the 
occurence of 'glancing points' that make the analysis much more delicate. This difficulty can be (formally) explained on the following simple example. Let $u$ satisfy

$$
\begin{cases}\left(i \partial_{t}+\Delta\right) u & =0,\left(x^{\prime}, x_{d}, t\right):=(x, t) \in \mathbb{R}^{d-1} \times \mathbb{R}^{+} \times \mathbb{R}, \\ \left.u\right|_{x_{d}=0} & =\varphi, \varphi \text { and all its derivatives vanishing at } t=0 .\end{cases}
$$

By Fourier-Laplace transform, we see that

$$
u\left(x^{\prime}, x_{d}, t\right)=\frac{1}{(2 \pi)^{d}} \iint_{\mathbb{R}^{d-1} \times \mathbb{R}} e^{\gamma t+i \delta t+i \xi x^{\prime}} e^{-\sqrt{i(\gamma+i \delta)-|\xi|^{2}} x_{d}} \widehat{\varphi}(\xi, \gamma+i \delta) d \xi d \delta
$$

If the dimension of space is 1 there is no $x^{\prime}$ and, letting $\gamma \rightarrow 0$ the integral splits

$$
u(x, t)=\frac{1}{2 \pi} \int_{\mathbb{R}^{+}} e^{i \delta t} e^{-i \sqrt{\delta} x} \widehat{\varphi}(i \delta) d \delta+\frac{1}{2 \pi} \int_{\mathbb{R}^{-}} e^{i \delta t} e^{-\sqrt{|\delta|} x} \widehat{\varphi}(i \delta) d \delta,
$$

from which the spacelike regularity of $u$ can be deduced: on the first integral, using a change of variable $\delta=\sigma^{2}$, we recognize a Fourier transform in $x$ (this nice idea originates at least to the seminal paper by Kenig, Ponce and Vega [14]), and it is easy to check that for fixed $t$, this integral defines a function in $H^{s}\left(\mathbb{R}^{+}\right)$if $\varphi \in H^{s / 2+1 / 4}\left(\mathbb{R}^{+}\right)$. The second integral may be controlled thanks to the exponential decrease, more specifically by application of a lemma (lemma 3.1) in [2]. If the dimension is greater than 1, the same kind of analysis can only be carried in the regions

$$
\mathcal{E}=\left\{(\delta, \xi):-\delta-|\xi|^{2}>0\right\}, \text { and } \mathcal{H}=\left\{(\delta, \xi):-\delta-|\xi|^{2}<0\right\},
$$

(they are usually called 'elliptic' and 'hyperbolic' regions). On the 'glancing' set $\{(\delta, \xi)$ : $\left.\delta+|\xi|^{2}=0\right\}$ (where 0 is a multiple root in $\zeta$ of $-\delta-|\xi|^{2}+\zeta^{2}$ ) there is neither exponential decrease of the integrand nor an oscillating phase, which is a clear obstruction to the derivation of a priori estimates.

For strictly hyperbolic first order systems, the same kind of technical difficulties was overcome by Kreiss [15] thanks to the construction of a tool now called Kreiss symmetrizer. Our aim here is to extend the analysis of Kreiss to more general, although constant coefficient, equations. In particular, while for hyperbolic equations it is convenient to work with the homogeneous principal part of the symbols, we shall work on quasi-homogeneous symbols.

More precisely, if $\alpha \in \mathbb{N}^{d}$ is an $n$-tuple we define $|\alpha|=\sum_{j=1}^{d} \alpha_{j}$. We say that a partial differential operator $P(\tau, \xi)$ is $p$-homogeneous of degree $m p$ if it has the form

$$
P\left(\partial_{t}, D_{x}\right)=\sum_{\substack{0 \leq j \leq m \\ p j+|\alpha|=m p}} a_{\alpha, j} D_{x}^{\alpha} \partial_{t}^{j}
$$

where $D_{x}^{\alpha}=\prod_{j=1}^{d} D_{j}^{\alpha_{j}}, D_{j}=\partial /\left(i \partial x_{j}\right)$. We define the $p$-degree of a monomial $D_{x}^{\alpha} \partial_{t}^{j}$ as $|\alpha|+p j$. The $p$-degree of a partial differential operator is the maximal $p$-degree of its 
monomials. If $P$ is a partial differential operator of $(p-)$ order $N$, its p-principal part is the operator obtained by keeping only terms of $p$-degree $N$, and we denote it by $\operatorname{princ}(P)$ if no confusion can occur regarding $p$. We say that an operator is resolved (in $\partial_{t}$ ) if its $p$-degree is a multiple of $p$, say $m p$, and the coefficient of $\partial_{t}^{m}$ is not 0 . In order to include simple equations like

$$
\partial_{t} u+c \cdot \nabla u+i \Delta u=0, c \in \mathbb{R}^{d},
$$

we will work on a larger class than $p$-homogeneous operators. Let $P\left(\partial_{t}, D_{x}\right)$ be a matrix of size $n$ of partial differential operators of order (at most) $m p$. We say that it is purely dispersive $^{1}$ if it is resolved and

$$
\sigma_{P}(\cdot, \xi):=\operatorname{det}(P(\cdot, \xi)) \text { has only purely imaginary roots for } \xi \in \mathbb{R}^{d} .
$$

The operator is strictly dispersive if

$P$ is purely dispersive and $\left.\operatorname{princ}\left(\sigma_{P}\right)(\cdot, \xi)\right)$ has distinct roots for $\xi \in \mathbb{R}^{d} \backslash\{0\}$.

Note that the idea of adaptating techniques from the hyperbolic field to quasi-homogeneous equations appeared rather early, notably with the work of Gindikin and Volevic [25]. In particular, they give a very nice discussion on the concept of symbols weakly correct in the sense of Petrowskii in their book [12], and find a class of scalar dispersive equations for which analysis of the mixed problem with variable coefficients is possible. Unfortunately this is a very special class (basically multidimensional generalizations of the Airy equation), and their analysis does not extend to systems.

Anisotropic weighted Sobolev spaces $H_{\gamma, p}^{s}$ and a generalization of the uniform KreissLopatinskil condition (UKL) are defined in section 1. Our main result concerns boundary value problem of the form

$$
\left\{\begin{array}{l}
P u=f \\
\left.B_{\gamma}\left(\partial_{t}, \partial_{x}\right) u\right|_{x_{d}=0}=\varphi
\end{array}\right.
$$

with $B_{\gamma}$ a boundary operator that we will be defined more precisely in section 1 .

Theorem 1. If $P=\sum_{\substack{\alpha, j \\ \mid \alpha+p j \leq m p}} A_{\alpha, j} D_{x}^{\alpha} \partial_{t}^{j}$ is strictly dispersive, the boundary is not characteristic, that is $A_{(0, \cdots, 0, m p), 0}$ is invertible, and $B_{\gamma}$ satisfies the uniform Kreiss-Lopatinskir condition, then there exists $\Gamma>0$ such that for any $\gamma \geq \Gamma$ and any

$$
(\varphi, f) \in H_{\gamma, p}^{\frac{p-1}{2}}\left(\mathbb{R}^{d-1} \times \mathbb{R}\right) \times H_{\gamma, p}^{p-1}\left(\mathbb{R}^{d-1} \times \mathbb{R}^{+} \times \mathbb{R}\right)
$$

\footnotetext{
${ }^{1}$ In not yet published lecture notes [http://www.math.lsa.umich.edu/ rauch/nlgonotes.pdf] , J. Rauch defines the pure dispersivity for homogeneous hyperbolic problems as a condition of non-flatness of the characteristic variety. This condition is fullfilled here because of the quasi-homogeneity.
} 
the problem (2) has an unique solution $u \in H_{\gamma, p}^{m p-1}$, and it satisfies the estimate

$$
\gamma\|u\|_{H_{\gamma, p}^{m p-1}}^{2}+\sum_{j=0}^{m p-1}\left|\partial_{x_{d}}^{j} u\right|_{H_{\gamma, p}^{m p-1-j+(p-1) / 2}}^{2} \leq C\left(\frac{1}{\gamma}\|f\|_{L^{2}\left(H_{\gamma, p}^{p-1}\right)}^{2}+|\varphi|_{H_{\gamma, p}^{(p-1) / 2}}^{2}\right),
$$

The proof of theorem 1 relies on the derivation of the a priori estimate (3) thanks to the construction of generalized Kreiss symmetrizers. The plan of this construction is similar to the classical one performed by Kreiss and explained in details in [6] or [18], but we have to deal with a new fact: lower order terms can not be neglected. This leads to a slight, but noticeable modification of the original construction. In particular it should be noted that the definition of a strictly dispersive operator involves the full symbol, and not only its $p$-homogeneous principal part.

Once the estimate is proved, we construct a dual boundary value problem, and finally prove the existence of a solution by standard Hahn-Banach arguments. The transition from boundary value problems to initial boundary value problems is rather delicate. In complete generality, we will only allow null initial data, but in the special case of the Schrödinger equation we will prove a well-posedness result for $H^{1}$ initial data and $H_{\gamma}^{3 / 2}\left(\mathbb{R}^{d-1} \times \mathbb{R}_{t}^{+}\right)$boundary data. To our knowledge, even for this simple case, this is a new result when $d>1$.

The paper is organized as follows.

- In section 1, we define a tangential differential calculus and functional spaces used in the article. The problem (2) is reformulated in the simpler form

$$
\partial_{x_{d}} U=G U+\tilde{f}
$$

with $G$ a tangential Fourier multiplier, and we define the uniform Kreiss Lopatinskil condition thanks to the analysis of the stable subspace of $G$.

- Section 2 is devoted to the derivation of a priori estimates for the boundary value problem. We introduce here the generalized Kreiss symmetrizers, whose lengthy and technical construction is postponed to the appendix.

- In section 3, we use a dual boundary value problem and the a priori estimate of section 2 to prove theorem 1 .

- In section 4, we deduce from these results well-posedness for zero initial data. For the non-homogeneous linear Schrödinger equation, the well-posedness of the mixed problem with $H^{1}$ initial data is also proved.

- In the appendix, we sketch the construction of generalized Kreiss symmetrizer, give references for the classical construction in the hyperbolic case and we explain what are the modifications needed to adapt it to our settings. 


\section{The Uniform Kreiss-Lopatinskiǔ condition}

Some notations From now on and until section 4 , we work on the pure boundary value problem posed on the half space $\mathbb{R}^{d-1} \times \mathbb{R}^{+} \times \mathbb{R}_{t}$. For clarity, we write $\mathbb{R}_{t}$ instead of $\mathbb{R}$ to insist on the fact that it corresponds to the time variable. Since the $x_{d}$ variable has a special role, we note $(x, t)=\left(x^{\prime}, x_{d}, t\right) \in \mathbb{R}^{d-1} \times \mathbb{R}^{+} \times \mathbb{R}_{t}$.

\section{Sobolev spaces and Fourier-Laplace multipliers}

Tangential operators For $\Omega$ an open set of $\mathbb{R}^{n}, n \geq 1$ and $\gamma \geq 0$ we introduce the weighted in time $L^{2}$ space

$$
\begin{aligned}
L_{\gamma}^{2}\left(\Omega \times \mathbb{R}_{t}\right):= & \left\{u \in L_{l o c}^{2}\left(\mathbb{R}_{t} ; L^{2}(\Omega)\right):\right. \\
& \left.\|u\|_{L_{\gamma}^{2}}^{2}=\|u\|_{\gamma}^{2}:=\iint_{\Omega \times \mathbb{R}_{t}} e^{-2 \gamma t}|u|^{2} d x d t<\infty\right\},
\end{aligned}
$$

and the tangential Fourier-Laplace transform

$$
\begin{array}{r}
u \in L_{\gamma}^{2}\left(\mathbb{R}^{d-1} \times \mathbb{R}^{+} \times \mathbb{R}_{t}\right) \rightarrow \widehat{u}, \\
\widehat{u}\left(\eta, x_{d}, \gamma+i \delta\right):=\iint_{\mathbb{R}^{d-1} \times \mathbb{R}^{+}} e^{-\gamma t-i \delta t-i \eta \cdot x^{\prime}} u\left(x^{\prime}, x_{d}, t\right) d x^{\prime} d t .
\end{array}
$$

For $\varphi$ defined on $\mathbb{R}^{d-1} \times \mathbb{R}_{t}$, we write in the same way $\widehat{\varphi}$ the usual Fourier-Laplace transform. A tangential Fourier multiplier $A^{\gamma}$ with parameter $\gamma \in \mathbb{R}^{+}$is defined by its symbol $a(\gamma+i \delta, \eta)$ and the formula

$$
\widehat{A^{\gamma} u}\left(\eta, x_{d}, i \delta\right):=a(\gamma+i \delta, \eta) \widehat{u}\left(\eta, x_{d}, i \delta\right)
$$

For such an operator, we use the notation $A_{\gamma}:=e^{\gamma t} A^{\gamma} e^{-\gamma t}$. In particular, we have the formula

$$
\widehat{A_{\gamma} u}\left(\eta, x_{d}, \gamma+i \delta\right)=a(\gamma+i \delta, \eta) \widehat{u}\left(\eta, x_{d}, \gamma+i \delta\right),
$$

and call $A_{\gamma}$ a tangential Fourier-Laplace multiplier, or for conciseness a Fourier multiplier. A Fourier multiplier will usually be denoted by a capital letter, and the corresponding small letter will be its symbol expressed in terms of $\gamma+i \delta$ and $\eta$. For $p \in \mathbb{N}, p \geq 2$, we denote by $\Lambda_{\gamma, p}:=e^{\gamma t} \Lambda_{p}^{\gamma} e^{-\gamma t}$ the tangential Fourier-Laplace multiplier of symbol

$$
\lambda_{p}(\gamma+i \delta, \eta):=\left(\gamma^{2}+\delta^{2}+|\eta|^{2 p}\right)^{1 /(2 p)} .
$$

We denote generically $\tau=\gamma+i \delta$ and say that the operator $A_{\gamma}$ is of $p$-order $n$ if its symbol $a(\tau, \eta)$ satisfies $|a(\tau, \eta)| \leq C \lambda_{p}^{n}$. It is quasi-homogeneous if for $r>0, a\left(r^{p} \tau, r \eta\right)=$ $r^{n} a(\tau, \eta)$. 
Finally let us point out the simple commutator identity for $L$ a constant coefficient differential operator

$$
\begin{array}{r}
L\left(\partial_{t}, D_{x^{\prime}}, \partial_{x_{d}}\right)\left(e^{\gamma t} u\right)=e^{\gamma t} L\left(\partial_{t}+\gamma, D_{x^{\prime}}, \partial_{x_{d}}\right) u, \\
\text { which implies } \widehat{L u}\left(\eta, x_{d}, \tau\right)=l\left(\tau, \eta, \partial_{x_{d}}\right) \widehat{u}\left(\eta, x_{d}, \tau\right),
\end{array}
$$

(here $l$ and $L$ are of course the same polynomial, but we distinguish the symbol from the operator).

In the rest of the paper, we will implicitly consider $\partial_{t}$ as an operator with parameter via the identification $\partial_{t}=e^{\gamma t}\left(\partial_{t}+\gamma\right) e^{-\gamma t}$.

Functional spaces We denote by $\mathcal{S}\left(\mathbb{R}^{d-1} \times \mathbb{R}^{+} \times \mathbb{R}_{t}\right)$ the set of restrictions on $\mathbb{R}^{d-1} \times \mathbb{R}^{+} \times \mathbb{R}_{t}$ of functions in $\mathcal{S}\left(\mathbb{R}^{d} \times \mathbb{R}_{t}\right)$ (Schwartz class of smooth and rapidly decaying functions), and $\mathcal{S}_{\gamma}:=\left\{u \in \mathcal{C}^{\infty}: e^{-\gamma t} u \in \mathcal{S}\left(\mathbb{R}^{d-1} \times \mathbb{R}^{+} \times \mathbb{R}_{t}\right)\right\}$. Their 'trace analogues' are denoted in the same way $\mathcal{S}_{\gamma}\left(\mathbb{R}^{d-1} \times \mathbb{R}_{t}\right)$.

For $s \geq 0$, we define the weighted anisotropic Sobolev spaces

$$
H_{\gamma, p}^{s}\left(\mathbb{R}^{d} \times \mathbb{R}_{t}\right):=\left\{u \in L_{\gamma}^{2}\left(\mathbb{R}^{d} \times \mathbb{R}_{t}\right): \iint_{\mathbb{R}^{d} \times \mathbb{R}}\left(|\tau|^{2}+|\xi|^{2 p}\right)^{2 n / p}|\widehat{u}(\xi, \tau)|^{2} d \xi d \delta<\infty\right\},
$$

and underline that for $r<s<t, H_{\gamma, p}^{s}$ can be seen as interpolated between $H_{\gamma, p}^{r}$ and $H_{\gamma, p}^{t}$. the spaces $H_{\gamma, p}^{s}\left(\mathbb{R}^{d-1} \times \mathbb{R}^{+} \times \mathbb{R}_{t}\right)$ are the spaces of restrictions of function in $H_{\gamma, p}^{s}\left(\mathbb{R}^{d} \times \mathbb{R}_{t}\right)$. If $s=n$ is an integer

$$
\begin{array}{r}
H_{\gamma, p}^{m}=\left\{u \in L_{\gamma}^{2}\left(\mathbb{R}^{d-1} \times \mathbb{R}^{+} \times \mathbb{R}_{t}\right):\right. \\
\left.\|u\|_{H_{\gamma, p}^{n}}^{2}:=\sum_{j=0}^{n} \iint_{\mathbb{R}^{d-1} \times \mathbb{R}^{+} \times \mathbb{R}_{t}} \lambda_{p}(\tau, \eta)^{2(n-j)}\left|\partial_{x_{d}}^{j} \widehat{u}(\xi, \tau)\right|^{2} d \xi d \delta<\infty\right\} .
\end{array}
$$

For $s \geq 0$ we denote the 'trace spaces':

$$
\begin{array}{r}
H_{\gamma, p}^{s}\left(\mathbb{R}^{d-1} \times \mathbb{R}_{t}\right):=\left\{\varphi \in L_{\gamma}^{2}\left(\mathbb{R}^{d-1} \times \mathbb{R}_{t}\right):|\varphi|_{H_{\gamma, p}^{s}}^{2}=|\varphi|_{s, \gamma, p}^{2}\right. \\
\left.:=\iint_{\mathbb{R}^{d-1} \times \mathbb{R}} \zeta_{p}^{2 s}|\widehat{\varphi}(\eta, \tau)|^{2} d \eta d \delta<\infty\right\} .
\end{array}
$$

In addition, $H_{0, \gamma, p}^{s}$ is defined as the closure of $\mathcal{C}_{c}^{\infty}$ functions for the $H_{\gamma, p}^{s}$ norm, and the negative index spaces are defined by duality $H_{\gamma, p}^{-s}=\left(H_{0, \gamma, p}^{s}\right)^{\prime}$. Clearly, any Fourier multiplier $A_{\gamma}$ of $p$-order $n$ acts continuously from $H_{\gamma, p}^{s}\left(\mathbb{R}^{d-1} \times \mathbb{R}_{t}\right)$ to $H_{\gamma, p}^{s-n}\left(\mathbb{R}^{d-1} \times \mathbb{R}_{t}\right)$. Finally, if $u$ belongs to some functional space $X\left(\mathbb{R}^{d-1} \times \mathbb{R}^{+} \times \mathbb{R}_{t}\right)$, we denote $\left.u\right|_{x_{d}=0}$ its trace (when it is defined), or $u(0)$ if there is no ambiguity. 


\section{Reformulated problem and stable subspaces}

Reformulation Let $u$ be a solution of (2) with

$$
\begin{array}{r}
P\left(\partial_{t}, D_{x}\right)=\sum_{k+|\alpha| \leq m p} A_{\alpha, k} D_{x}^{\alpha} \partial_{t}^{k}=\sum_{j=0}^{m p} A_{m p-j}^{\prime}\left(\partial_{t}, D_{x^{\prime}}\right) \partial_{x_{d}}^{j} \\
B_{\gamma}\left(\partial_{t}, D_{x^{\prime}}, \partial_{x_{d}}\right)=\sum_{j=0}^{m p-1} B_{m p-1-j, \gamma}\left(\partial_{t}, D_{x^{\prime}}\right) \partial_{x_{d}}^{j}
\end{array}
$$

where $B_{k, \gamma}$ is a Fourier multiplier of order $k$ and $A_{k}^{\prime}$ a constant coefficient differential operator of order $k$ and size $n$. Since we deal with noncharacteristic problems, we may assume $A_{0}^{\prime}=I_{n}$ (note that the multiplication of the system by a constant matrix does not change the hypothesis $(D s))$. Let us introduce the new unknown

$$
\widetilde{u}:=\left(\begin{array}{c}
\Lambda_{\gamma, p}^{m p-1} u \\
\Lambda_{\gamma, p}^{m p-2} \partial_{x_{d}} u \\
\vdots \\
\partial_{x_{d}}^{m p-1} u
\end{array}\right)
$$

then $\widetilde{u}$ satisfies

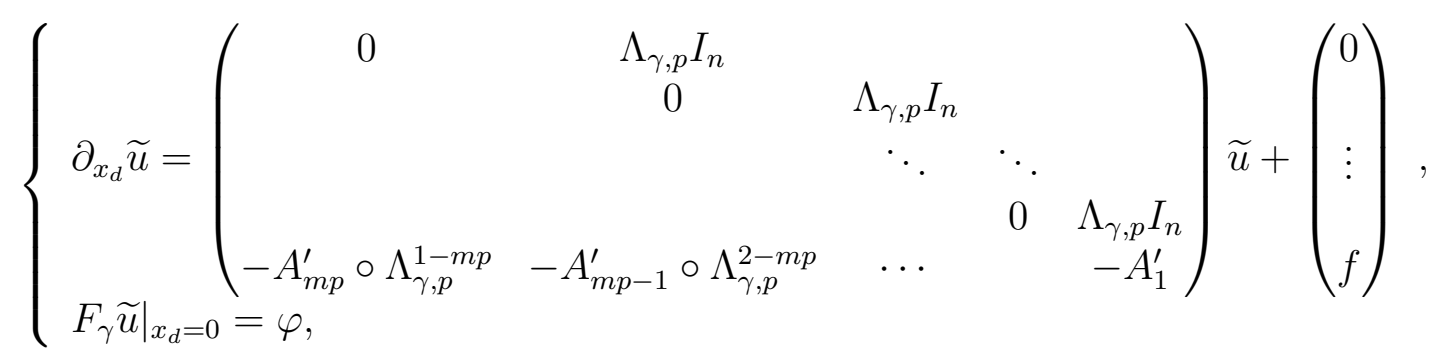

with $F_{\gamma}:=\left(\begin{array}{llll}\left(B_{m p-1}\left(\Lambda_{p}^{\gamma}\right)^{1-m p}\right)_{\gamma} & \left(B_{m p-2}\left(\Lambda_{p}^{\gamma}\right)^{2-m p}\right)_{\gamma} & \cdots & B_{0, \gamma}\end{array}\right)$.

Using (6), we have for $0 \leq j \leq m p-1$

$$
A_{j}^{\prime}\left(\partial_{t}, D_{x^{\prime}}\right) \Lambda_{\gamma, p}^{1+j-m p}=\left(A_{j}^{\prime}\left(\partial_{t}+\gamma, D_{x^{\prime}}\right)\left(\Lambda_{p}^{\gamma}\right)^{1+j-m p}\right)_{\gamma}
$$

thus the problem (11) reads

$$
\left\{\begin{array}{l}
\partial_{x_{d}} \widetilde{u}=G_{\gamma} U+f^{\prime}:=G_{\gamma} \widetilde{u}+\left(\begin{array}{c}
0 \\
\vdots \\
f
\end{array}\right), \\
\left.F_{\gamma} \widetilde{u}\right|_{x_{d}=0}=\varphi,
\end{array}\right.
$$


with $G_{\gamma}\left(\right.$ resp. $\left.F_{\gamma}\right)$ a Fourier multiplier of order 1 (resp. 0), and of symbol

$$
\begin{aligned}
& g(\tau, \eta):=\left(\begin{array}{ccccc}
0 & \lambda_{p} & & & \\
0 & \lambda_{p} & & \\
& & \ddots & \ddots & \\
-\left(a_{m p}^{\prime} \lambda_{p}^{1-m p}\right)(\tau, \eta) & -\left(a_{m p-1}^{\prime} \lambda_{p}^{2-m p}\right)(\tau, \eta) & \cdots & & -a_{1}^{\prime}(\tau, \eta)
\end{array}\right), \\
& F(\tau, \eta):=\left(\begin{array}{llll}
b_{m p-1} \lambda_{p}^{1-m p} & b_{m p-2} \lambda_{p}^{2-m p} & \cdots & b_{0}
\end{array}\right)(\tau, \eta) .
\end{aligned}
$$

We write $F$ for the symbol of $F$ instead of $f$ in order to avoid any confusion with the forcing term. Since $g$ is a companion matrix, we have the identity

$$
\left.\chi_{g(\tau, \eta)}(\zeta):=\operatorname{det}\left(g(\tau, \eta)-\zeta I_{m p n}\right)\right)=\sigma_{P}(\tau, \eta, \zeta / i) .
$$

By Fourier-Laplace transform, the problem rewrites for $U=\widehat{\widetilde{u}}$ and fixed $\gamma:=\operatorname{Re}(\tau)>0$ as a family of ordinary differential equations

$$
\forall(\tau, \eta) \in\{\operatorname{Re}(\tau)=\gamma\} \times \mathbb{R}^{d-1},\left\{\begin{array}{l}
\partial_{x_{d}} U=g(\tau, \eta) U+\widehat{f}^{\prime}, \\
\left.F(\tau, \eta) U\right|_{x_{d}=0}=\widehat{\varphi}, \\
\lim _{x_{d} \rightarrow+\infty} U\left(\eta, x_{d}, \tau\right)=0 .
\end{array}\right.
$$

The last condition comes from the fact that in view of theorem 1 we look for square integrable solutions. If we denote by $E^{-}(g(\tau, \eta))$ the stable subspace of $g$, that is the space of vectors $X$ such that

$$
\left|e^{g x_{d}} X\right| \rightarrow_{x_{d} \rightarrow+\infty} 0,
$$

the system above simply rewrites

$$
\forall(\tau, \eta) \in\{\operatorname{Re}(\tau)=\gamma\} \times \mathbb{R}^{d-1},\left\{\begin{array}{l}
\partial_{x_{d}} U=g(\tau, \eta) U+\widehat{f}^{\prime} \\
\left.F(\tau, \eta) U\right|_{x_{d}=0}=\widehat{\varphi} \\
\left.U\right|_{x_{d}=0} \in E^{-}(g)
\end{array}\right.
$$

The Kreiss-Lopatinskiur condition The previous analysis motivates the following definition.

Definition 1. We say that the boundary operator $F$ of (12) satisfies the generalized Kreiss-Lopatinskiu condition if there exists $\Gamma>0$ such for any $(\tau, \eta) \in \mathcal{E}^{\Gamma}:=\{\operatorname{Re}(\tau) \geq$ $\Gamma\} \times \mathbb{R}^{d-1}, F(\tau, \eta): E^{-}(g(\tau, \eta)) \rightarrow \operatorname{Im}(F)$ is an isomorphism.

This condition is said to be uniform when $F$ and $F^{-1}: \operatorname{Im}(F) \rightarrow E^{-}$are uniformly bounded for $(\tau, \eta) \in\left\{\operatorname{Re}(\tau \geq \Gamma\} \times \mathbb{R}^{d-1}\right.$. We refer to it as (KLU), and since $F$ is defined thanks to $B_{\gamma}$ of (2), we say that $B_{\gamma}$ satisfies the (uniform) Kreiss-Lopatinskir condition if $F$ does. 
The Kreiss-Lopatinskiǔ condition is well known for hyperbolic boundary value problems since the work of Kreiss [15]. It is a necessary condition of well-posedness: if a boundary value problem does not meet it, one can show (see [1] prop. 4.2 p.107 for example) that it is ill-posed in the sense of Hadamard. In the hyperbolic frame there are various stronger versions of this hypothesis implying well-posedness (possibly with loss of derivatives, see M. Sablé-Tougeron [22] or J-F. Coulombel [7]). The so-called uniform Kreiss-Lopatinskiu condition is the strongest one and Kreiss proved that for strictly hyperbolic problems it implies well-posedness as well as a priori estimates without loss of derivatives.

In the rest of this section, we focus on the derivation of a simpler definition for (KLU) thanks to the study of $E^{-}(g)$.

Continuation of the stable subspace We first recall the Dunford-Taylor formula: for a matrix $A$, if $C$ is a contour of a part of $A$ 's spectrum $\operatorname{Sp}(A), C \cap \operatorname{Sp}(A)=\emptyset$, then the projector on the corresponding eigenspace is given by

$$
\Pi=\frac{1}{2 i \pi} \int_{C}(A-z I)^{-1} .
$$

Proposition 1. If the operator $P$ in (8) is purely dispersive (as defined in (Dp) p.3), then for any $(\tau, \eta) \in \mathcal{E}^{++}:=\{\operatorname{Re}(\tau)>0\} \times \mathbb{R}^{d-1}, g(\tau, \eta)$ has no purely imaginary eigenvalue. In particular, $E^{-}(g)$ is exactly the sum of eigenspaces associated to eigenvalues of negative real parts and its dimension on $\mathcal{E}^{++}$remains constant.

Proof. According to (13), the characteristic polynomial of $g$ is $\sigma_{P}(\tau, \eta, \zeta / i)$. If $\zeta$ is a purely imaginary eigenvalue, then $\operatorname{Re}(\tau)=0$ since $P$ is purely dispersive, and thus there is no purely imaginary eigenvalue when $\operatorname{Re}(\tau)>0$. By continuity in $(\tau, \eta)$ of the rooths of $P(\tau, \eta, \cdot)$ and connexity of $\mathcal{E}^{++}$, we find that the number of roots of negative real part remains constant on $\mathcal{E}^{++}$, and so does $\operatorname{dim}\left(E^{-}\right)$.

According to the Dunford-Taylor formula, the projectors on $E^{-}$are $\mathcal{C}^{\infty}$ on $\mathcal{E}^{++}$, and thus $E^{-}$defines (the fibers of) a $C^{\infty}$ vector bundle of base space $\mathcal{E}^{++}$. An essential point is the continuous extension of this bundle on a larger base. We first introduce some convenient notations. Similarly to $\mathcal{E}^{++}$, we note $\mathcal{E}^{+}:=\left\{(\tau, \eta) \in \mathbb{C} \times \mathbb{R}^{d-1}: \operatorname{Re}(\tau) \geq 0\right\}$. For $(\tau, \eta)$ such that $\lambda_{p}(\tau, \eta) \geq 1$, the 'radial compactification'

$$
(\tau, \eta) \rightarrow\left(\tau / \lambda_{p}^{p}, \eta / \lambda_{p}, 1 / \lambda_{p}\right)=:(\widehat{\tau}, \widehat{\eta}, \varepsilon)
$$

defines a diffeomorphism

$$
\begin{aligned}
\mathcal{E}^{+} \cap\left\{\lambda_{p}(\tau, \eta) \geq 1\right\} & \rightarrow\left\{(\widehat{\tau}, \widehat{\eta}, \varepsilon): \operatorname{Re}(\widehat{\tau}) \geq 0,\left(|\widehat{\tau}|^{2}+|\widehat{\eta}|^{2 p}\right)^{1 /(2 p)}=1,0<\varepsilon \leq 1\right\} \\
& \left.\left.=: \mathbb{S}_{p}^{+} \times\right] 0,1\right]
\end{aligned}
$$


For $(\tau, \eta)$ such that $\lambda(\tau, \eta) \geq 1$ and $\psi$ a function of $(\tau, \eta)$, we will (abusively) write $\psi(\tau, \eta)=\psi(\widehat{\tau}, \widehat{\eta}, \varepsilon)$. Since the radial compactification is a diffeomorphism, $\psi(\tau, \eta)$ and $\psi(\widehat{\tau}, \widehat{\eta}, \varepsilon)$ have the same level of regularity. Finally, we underline that $h(\widehat{\tau}, \widehat{\eta}, \varepsilon)=$ $h(\tau, \eta):=g(\tau, \eta) / \lambda_{p}(\tau, \eta)$ is $\mathcal{C}^{\infty}$ on $\mathbb{S}_{p}^{+} \times[0,1]$ with, if princ denotes the $p$ principal part as defined in the introduction,

$$
h(\widehat{\tau}, \widehat{\eta}, 0)=\left(\begin{array}{ccccc}
0 & 1 & & & \\
& 0 & 1 & & \\
& & \ddots & \ddots & \\
-\operatorname{princ}\left(a_{m p}^{\prime}\right)(\widehat{\tau}, \widehat{\eta}) & -\operatorname{princ}\left(a_{m p-1}^{\prime}\right)(\widehat{\tau}, \widehat{\eta}) & \cdots & & -\operatorname{princ}\left(a_{1}^{\prime}\right)
\end{array}\right) .
$$

We have $E^{-}(g(\tau, \eta))=E^{-}(h(\tau, \eta))$ thus we may simply write $E^{-}(\tau, \eta)$ with no risk of confusion. The characteristic polynomial of $h=\varepsilon g(\widehat{\tau}, \widehat{\eta}, \varepsilon)$ is

$$
\chi_{h(\widehat{\tau}, \widehat{\eta}, \varepsilon)}(\zeta)=\varepsilon^{m p n} \sigma_{P}\left(\frac{\widehat{\tau}}{\varepsilon^{p}}, \frac{\widehat{\eta}}{\varepsilon}, \frac{\zeta}{i \varepsilon}\right),
$$

which is polynomial in $(\widehat{\tau}, \widehat{\eta}, \varepsilon, \zeta)$. In particular it is analytic even for $\varepsilon=0$, where $\chi_{h(\widehat{\tau}, \widehat{\eta}, 0)}(\zeta)=\operatorname{princ}\left(\sigma_{P}\right)(\widehat{\tau}, \widehat{\eta}, \zeta / i)$.

From now on, we will state our results in the frame of the more convenient variables $(\widehat{\tau}, \widehat{\eta}, \varepsilon)$, although we are interested in their immediate counterpart for $(\tau, \eta)$. Note that an immediate adaptation of Proposition 1 above shows that $\chi_{h(\widehat{\tau}, \widehat{\eta}, 0)}(\cdot)$ has no purely imaginary root if $\operatorname{Re}(\widehat{\tau})>0$, thus $E^{-}(h)$ has a constant rank and is smoothly defined on the connected set $\mathbb{S}_{p}^{++} \times[0,1]$. We start with the following elementary but useful lemma.

Lemma 1. If $P\left(\partial_{t}, D_{x}\right)$ is strictly dispersive, there exists $C>0$ such that

$$
\begin{array}{r}
\forall(\widehat{\tau}, \widehat{\xi}, \varepsilon) \in \mathbb{C} \times \mathbb{R}^{d} \times[0,1],\left(|\widehat{\tau}|^{2}+|\widehat{\xi}|^{2 p}\right)^{1 /(2 p)}=1, \varepsilon \leq 1 / C, \\
\varepsilon^{m n p} \sigma_{P}\left(\frac{\widehat{\tau}}{\varepsilon}, \frac{\widehat{\xi}}{\varepsilon}\right)=0 \Rightarrow \widehat{\tau} \text { is a simple root of } \varepsilon^{m n p} \sigma_{P}\left(\frac{\cdot}{\varepsilon}, \frac{\widehat{\xi}}{\varepsilon}\right) .
\end{array}
$$

Proof. Let us assume that there exists a sequence $\left(\widehat{\tau_{k}}, \widehat{\xi_{k}}, \varepsilon_{k}\right)$ with $\varepsilon_{k} \rightarrow 0$ contradicting the result. The sequence $\left(\widehat{\tau_{k}}, \widehat{\xi_{k}}\right)$ is bounded and we may extract a converging subsequence of limit $(\widehat{\tau}, \widehat{\xi}) \neq 0$. But since

$$
\varepsilon_{k}^{n m p}\left(\sigma_{P}\right)\left(\frac{\cdot}{\varepsilon_{k}}, \frac{\dot{ }}{\varepsilon_{k}}\right) \rightarrow \operatorname{princ}\left(\sigma_{P}\right)(\cdot, \cdot) \text { uniformly, }
$$

we find that $\widehat{\tau}$ is a multiple root of $\operatorname{princ}\left(\sigma_{P}\right)(\cdot, \xi)$, thus the strict dispersivity implies $\widehat{\xi}=0$ and since the polynomial is resolved we have $\widehat{\tau}=0$. This is a contradiction. 
Proposition 2. Let $\left(\zeta_{j}\right)$ be the roots of the characteristic polynomial of $h(\widehat{\tau}, \widehat{\eta}, \varepsilon)$.

The application $\sigma_{+}$defined by

$$
\forall(\widehat{\tau}, \widehat{\eta}, \varepsilon) \in \mathbb{S}_{p}^{++} \times[0,1], \sigma_{+}(\zeta ; \widehat{\tau}, \widehat{\eta}, \varepsilon):=\prod_{\operatorname{Re}\left(\lambda_{j}(\widehat{\tau}, \widehat{\eta}, \varepsilon)\right)>0}\left(\zeta-\zeta_{j}\right)
$$

has a continuous extension on $\mathbb{S}_{p}^{+} \times[0,1]$. If $C$ is the constant of Lemma 1 and $P$ is strictly dispersive, then

$$
E^{-}(\widehat{\tau}, \widehat{\eta}, \varepsilon)=\operatorname{Im}\left(\sigma_{+}(g(\widehat{\tau}, \widehat{\eta}, \varepsilon) ; \widehat{\tau}, \widehat{\eta}, \varepsilon)\right)
$$

defines a continuous extension of $E^{-}$on $\mathbb{S}_{p}^{+} \times[0,1 / C]$.

Remark 3. Roughly speaking, this proposition gives a control of $E^{-}(\tau, \eta)$ for high frequencies.

Proof. The characteristic polynomial of $h=\varepsilon g(\widehat{\tau}, \widehat{\eta}, \varepsilon)$ is

$$
\varepsilon^{m p n} \sigma_{P}\left(\frac{\widehat{\tau}}{\varepsilon^{p}}, \frac{\widehat{\eta}}{\varepsilon}, \frac{\zeta}{i \varepsilon}\right),
$$

polynomial in $(\widehat{\tau}, \widehat{\eta}, \varepsilon, \zeta)$ and in particular smooth on $\mathbb{S}_{p}^{+} \times[0,1]$. Thus the roots $\lambda_{j}$ are continuous functions of $(\widehat{\tau}, \widehat{\eta}, \varepsilon)$ on this set and $\sigma_{+}$can be continuously extended on $\mathbb{S}_{p}^{+} \times[0,1]$ simply by density of $\mathbb{S}_{p}^{++} \times[0,1]$ in $\mathbb{S}_{p}^{+} \times[0,1]$. If $\operatorname{Re}(\widehat{\tau})>0$ there is no purely imaginary roots, $\chi_{h(\widehat{\tau}, \widehat{\eta}, \varepsilon)}(\zeta)=\sigma_{+}(\zeta ; \widehat{\tau}, \widehat{\eta}, \varepsilon) \sigma_{-}(\zeta ; \widehat{\tau}, \widehat{\eta}, \varepsilon)$ and $\sigma_{-}$has only roots in $\zeta$ of negative real part. By the Hamilton-Cayley theorem, $E^{-}(\widehat{\tau}, \widehat{\eta}, \varepsilon)=\operatorname{Ker}\left(\sigma_{-}(g)\right)$, this implies then $E^{-}(\widehat{\tau}, \widehat{\eta}, \varepsilon)=\operatorname{Im}\left(\sigma_{+}\right)$.

Since $\sigma_{+}(h)$ is continuous on $\mathbb{S}_{p}^{+} \times[0,1]$, the continuity of the bundle $E^{-}$on $\mathbb{S}_{p}^{+} \times[0,1 / C]$ is equivalent to the fact that $\sigma_{+}(h)$ has a constant rank on $\mathbb{S}_{p}^{+} \times[0,1 / C]$. On $\mathbb{S}_{p}^{++} \times[0,1 / C]$ this is an immediate consequence of the absence of purely imaginary roots (Prop. 2). If $\left(\widehat{\tau_{0}}, \widehat{\eta}_{0}, \varepsilon_{0}\right) \in \mathbb{S}_{p}^{+} \times[0,1 / C]$ with $\operatorname{Re}\left(\widehat{\tau_{0}}\right)=0$, we denote

- The number $m^{ \pm}$of eigenvalues of $h\left(\widehat{\tau_{0}}, \widehat{\eta_{0}}, \varepsilon_{0}\right)$ of positive/negative real part,

- The purely imaginary eigenvalues $\left(i \omega_{1}, \cdots, i \omega_{r}\right)$ of $h\left(\widehat{\tau_{0}}, \widehat{\eta_{0}}, \varepsilon_{0}\right)$, and $m_{j}$ their algebraic multiplicity.

- The number $m_{j}^{-}$of eigenvalues of $h\left(\widehat{\tau_{0}}+\widehat{\gamma}, \widehat{\eta_{0}}, \varepsilon_{0}\right)$ of negative real part that have limit $i \omega_{j}$ when $\widehat{\gamma} \rightarrow 0$.

With these notations, we have clearly

$$
\mu:=\operatorname{dim}\left(E^{-}\left(\widehat{\tau_{0}}+\widehat{\gamma}, \widehat{\eta_{0}}, \varepsilon_{0}\right)\right)=m^{-}+\sum_{j=1}^{r} m_{j}^{-},
$$


and the operator $\sigma_{+}(h)$ has a constant rank if $\mu$ is the rank of

$$
\sigma_{+}\left(h, \widehat{\tau_{0}} ; \widehat{\eta_{0}}, \varepsilon_{0}\right)=\prod_{\operatorname{Re}\left(\zeta_{j}\right)>0}\left(h\left(\widehat{\tau_{0}}, \widehat{\eta_{0}}, \varepsilon_{0}\right)-\zeta_{j}\right) \prod_{j=1}^{r}\left(h\left(\widehat{\tau_{0}}, \widehat{\eta_{0}}, \varepsilon_{0}\right)-i \omega_{j}\right)^{m_{j}-m_{j}^{-}}
$$

After Jordan reduction one may see that that it is equivalent to the fact that every $i \omega_{j}$ has geometric multiplicity 1, i.e. is only associated to one Jordan block.

We prove it by contradiction: if for some $j, i \omega_{j}$ has a geometric multiplicity greater than 2 , then there is a change of basis such that $h_{j}$ reduces as

$$
P^{-1} h_{j}\left(\widehat{\tau_{0}}, \widehat{\eta}_{0}, \varepsilon_{0}\right) P=\left(\begin{array}{ccc}
J_{k_{1}}\left(i \omega_{j}\right) & 0 & 0 \\
0 & J_{k_{2}}\left(i \omega_{j}\right) & 0 \\
0 & 0 & \star
\end{array}\right)
$$

with $J_{k_{i}}$ two Jordan blocks. Now since

$$
\operatorname{det}\left(P^{-1} h_{j}\left((\widehat{\tau}, \widehat{\eta}, \varepsilon)-i \omega_{j}\right) P\right)=\chi_{h(\widehat{\tau}, \widehat{\eta}, \varepsilon)}\left(i \omega_{j}\right)=\varepsilon^{m n p} \sigma_{P}\left(\frac{\widehat{\tau}}{\varepsilon^{p}}, \frac{\widehat{\eta}}{\varepsilon}, \frac{\omega_{j}}{\varepsilon}\right),
$$

we find by differentiation and by using (17) that

$$
\frac{\partial \sigma_{P}}{\partial \widehat{\tau}}\left(\frac{\widehat{\tau_{0}}}{\varepsilon_{0}^{p}}, \frac{\widehat{\eta}_{0}}{\varepsilon_{0}}, \frac{\omega_{j}}{\varepsilon_{0}}\right)=0 \Leftrightarrow \widehat{\tau_{0}} / \varepsilon_{0}^{p} \text { is a multiple root of } \sigma_{P}\left(\cdot, \frac{\widehat{\eta_{0}}}{\varepsilon_{0}}, \frac{\omega_{j}}{\varepsilon_{0}}\right),
$$

which is a contradiction with Lemma 1.

As a first application of these results, we give a simpler form of (KLU).

Proposition 4. Let $E^{-}$be the stable subspace of $g$, extended by Prop. 2 on $\mathbb{S}_{p}^{+} \times[0,1 / C]$. The condition (KLU) (Definition 1 p.8) is true for some $\Gamma>0$ if and only if for any $(\widehat{\tau}, \widehat{\eta}) \in \mathbb{S}_{p}^{+}, F$ is an isomorphism $E^{-}(\widehat{\tau}, \widehat{\eta}, 0) \rightarrow \operatorname{Im}(F)$.

Proof. We first check the necessity: (KLU) means that $F: E^{-} \rightarrow \operatorname{Im}(F)$ and its inverse are uniformly bounded for $(\tau, \eta) \in\{\operatorname{Re}(\tau) \geq \Gamma\} \times \mathbb{R}^{d-1}$. The 'radial compactification' of this set is $\left.\left.\Omega:=\left\{(\widehat{\tau}, \widehat{\eta}, \varepsilon) \in \mathbb{S}_{p}^{+} \times\right] 0,1 / \Gamma\right]: \operatorname{Re}(\widehat{\tau}) \geq \varepsilon^{p} \Gamma\right\}$. By uniform boundedness, $F$ is invertible on the closure of $\Omega$, and since $\bar{\Omega} \supset \mathbb{S}_{p}^{+} \times\{0\}$, this proves the necessity.

Conversely, if $F(\widehat{\tau}, \widehat{\eta}, 0)$ is an isomorphism for $(\widehat{\tau}, \widehat{\eta}) \in \mathbb{S}_{p}^{+}$, then by (lower semi-) continuity there exists $\varepsilon_{0}$ small enough such that it is still an isomorphism for $(\widehat{\tau}, \widehat{\eta}, \varepsilon) \in \mathbb{S}_{p}^{+}\left[0, \varepsilon_{0}\right]$. By compacity, $F^{-1}$ is uniformly bounded on this set, which implies that $F^{-1}(\tau, \eta)$ is uniformly bounded for $\operatorname{Re}(\tau) \geq 1 / \varepsilon_{0}$. 


\section{$2 \quad$ Kreiss symmetrizers and a priori estimates}

We define here the generalized Kreiss symmetrizers and show how their existence implies a priori estimates without loss of derivatives.

Definition 2. A generalized Kreiss symmetrizer for the boundary value problem (12) is a Fourier multiplier $S_{\gamma}$ of symbol $s(\tau, \eta)$ defined and smooth on $\mathcal{E}^{\gamma}$ for some $\Gamma>0$. It satisfies

- The operator $S$ is of p-order 0 and for any $(\tau, \eta) \in \mathcal{E}_{\Gamma}, s^{*}=s$,

- There exists $c(\tau, \eta)>0$ such that (in the sense of the usual scalar product)

$$
\forall(\tau, \eta) \in \mathcal{E}^{\Gamma}, \operatorname{Re}(s g(\tau, \eta)):=\frac{s g+(s g)^{*}}{2} \geq c(\tau, \eta) \operatorname{Re}(\tau)
$$

- There exists $C>0$ such that

$$
\forall(\tau, \eta) \in \mathcal{E}^{\Gamma}, s(\tau, \eta) \geq I-C F^{*} F(\tau, \eta)
$$

Theorem 2. Let $P$ be a strictly dispersive operator, let (12) be the first order boundary value problem obtained from (2). If the boundary operator F satisfy (KLU) (note that $(K L U)$ depends on $G$ ) then there exists a generalized Kreiss symmetrizer for (12) with $c(\tau, \eta)=\alpha / \lambda_{p}^{p-1}, \alpha>0$.

If moreover, the spatial part of $P$ is elliptic, that is

$$
\forall \xi \in \mathbb{R}^{d} \backslash\{0\}, \sigma_{P}(0, \xi) \neq 0,
$$

then there exists a symmetrizer with $c(\tau, \eta)=\alpha /|\tau|^{(p-1) / p}$.

Remark 5. The usual Kreiss symmetrizers have homogeneous symbols of degree 0, and they satisfy $s g \geq \alpha \operatorname{Re}(\tau)$. The fact that here the lower bound goes to 0 as $(\tau, \eta) \rightarrow \infty$ is not a technical weakness of the construction but a natural (and unavoidable) fact due to the dispersive nature of the equations. The analysis in appendix should clarify it.

We postpone the (sketch of) proof of this result to the appendix. The point of generalized Kreiss symmetrizer lies in the following result.

Theorem 3. If the boundary value problem (12) admits a generalized Kreiss symmetrizer, then any $\widetilde{u} \in L_{\gamma}^{2}$ solution of

$$
\left\{\begin{array}{l}
\partial_{x_{d}} \widetilde{u}-G_{\gamma} \widetilde{u}=f^{\prime} \in L^{2}\left(\mathbb{R}^{+} ; H_{\gamma, p}^{p-1}\left(\mathbb{R}^{d-1} \times \mathbb{R}_{t}\right)\right) \\
\left.\left(F_{\gamma} \widetilde{u}\right)\right|_{x_{d}=0}=\varphi \in H_{\gamma, p}^{\frac{p-1}{2}}\left(\mathbb{R}^{d-1} \times \mathbb{R}_{t}\right) .
\end{array}\right.
$$


admits a trace on $\left\{x_{d}=0\right\}$ that is $H_{\gamma, p}^{\frac{p-1}{2}}$ and $\widetilde{u}$ satisfies the estimate

$$
\begin{gathered}
\forall \gamma \geq \Gamma, \gamma\|\widetilde{u}\|_{L_{\gamma}^{2}\left(\mathbb{R}^{d-1} \times \mathbb{R}^{+} \times \mathbb{R}_{t}\right)}^{2}+|\widetilde{u}(0)|_{H_{\gamma, p}^{(p-1) / 2}\left(\mathbb{R}^{d-1} \times \mathbb{R}_{t}\right)}^{2} \\
\lesssim \frac{1}{\gamma}\left\|\partial_{x_{d}} \widetilde{u}-G_{\gamma} \widetilde{u}\right\|_{L^{2}\left(\mathbb{R}^{+} ; H_{\gamma, p}^{p-1}\left(\mathbb{R}^{d-1} \times \mathbb{R}_{t}\right)\right)}^{2}+|F \widetilde{u}(0)|_{H_{\gamma, p}^{(p-1) / 2}\left(\mathbb{R}^{d-1} \times \mathbb{R}_{t}\right)}^{2}
\end{gathered}
$$

Moreover, if $\widetilde{u} \in \mathcal{S}_{\gamma}$, we have for any $s \in \mathbb{R}$,

$$
\begin{aligned}
& \forall \gamma \geq \Gamma, \gamma\|\widetilde{u}\|_{L^{2}\left(\mathbb{R}^{+} ; H_{\gamma, p}^{s}\right)}^{2}+|\widetilde{u}(0)|_{L^{2}\left(\mathbb{R}^{+} ; H_{\gamma, p}^{s+(p-1) / 2}\right)}^{2} \\
& \lesssim \frac{1}{\gamma}\left\|\partial_{x} \widetilde{u}-G_{\gamma} \widetilde{u}\right\|_{L^{2}\left(\mathbb{R}^{+} ; H_{\gamma, p}^{s+p-1}\right)}^{2}+|F \widetilde{u}(0)|_{H_{\gamma, p}^{s+(p-1) / 2}}^{2}
\end{aligned}
$$

If the symbol $P$ (or equivalently the characteristic polynomial of $g$, according to (13)) is elliptic in space, then under the same hypotheses the estimates become

$$
\begin{gathered}
\forall \gamma \geq \Gamma, \gamma\|\widetilde{u}\|_{L_{\gamma}^{2}\left(\mathbb{R}^{d-1} \times \mathbb{R}^{+} \times \mathbb{R}_{t}\right)}^{2}+|\widetilde{u}(0)|_{L^{2}\left(\mathbb{R}^{d-1} ; H_{\gamma}^{(p-1) /(2 p)}\left(\mathbb{R}_{t}\right)\right)}^{2} \\
\lesssim \frac{1}{\gamma}\left\|\partial_{x_{d}} \widetilde{u}-G \widetilde{u}\right\|_{L^{2}\left(\mathbb{R}^{d-1} \times \mathbb{R}^{+} ; H_{\gamma}^{(p-1) / p}\left(\mathbb{R}_{t}\right)\right)}^{2}+|F \widetilde{u}(0)|_{L^{2}\left(\mathbb{R}^{d-1} ; H_{\gamma}^{(p-1) /(2 p)}\left(\mathbb{R}_{t}\right)\right)}^{2}
\end{gathered}
$$

and

$$
\begin{array}{r}
\forall \gamma \geq \Gamma, \gamma\|\widetilde{u}\|_{L^{2}\left(\mathbb{R}^{d-1} \times \mathbb{R}^{+} ; H_{\gamma}^{s}\left(\mathbb{R}_{t}\right)\right)}^{2}+|\widetilde{u}(0)|_{L^{2}\left(\mathbb{R}^{d-1} \times \mathbb{R}^{+} ; H_{\gamma}^{(s+(p-1) / 2) / p}\left(\mathbb{R}_{t}\right)\right)}^{2} \\
\lesssim \frac{1}{\gamma}\left\|\partial_{x} \widetilde{u}-G \widetilde{u}\right\|_{L^{2}\left(\mathbb{R}^{d-1} \times \mathbb{R}^{+} ; H_{\gamma}^{(s+p-1) / p}\left(\mathbb{R}_{t}\right)\right)}^{2}+|F \widetilde{u}(0)|_{L^{2}\left(\mathbb{R}^{d-1} \times \mathbb{R}^{+} ; H_{\gamma}^{(s+(p-1) / 2) / p}\left(\mathbb{R}_{t}\right)\right)}^{2}(
\end{array}
$$

Remark 6 . Note that in the 'elliptic' case, there is additional regularity at the boundary only for the variable $t$, and the Sobolev spaces $H_{\gamma, p}^{s+(p-1) / 2}$ become $L^{2}\left(H_{\gamma}^{(p-1) /(2 p)}\right)$ without $p$ in index, since the anisotropic spaces have $p$ times more regularity in space than in time.

An other version of $E D e l l, s)$ may be given with $s$ additional regularity in $x^{\prime}$ variables too, we did not include it in order to shorten the (already tedious) list of estimates to come.

Proof. As a first step we apply formally an energy method. We have by Fourier-Laplace transform

$$
\partial_{x_{d}} U-g U=\widehat{f}^{\prime} .
$$

If we multiply this identity by the symbol $s$ of the symmetrizer, take the inner product with $U$, integrate in $x_{d}$ and take the real part we obtain

$$
\begin{array}{r}
\langle s U(\eta, 0, \tau), U(\eta, 0, \tau)\rangle+\operatorname{Re}\left(\int_{0}^{\infty}\left\langle s g U\left(\eta, x_{d}, \tau\right), U\left(\eta, x_{d}, \tau\right)\right\rangle d x_{d}\right) \\
=-\operatorname{Re}\left(\int_{0}^{\infty}\left\langle s \widehat{f}^{\prime}, U\left(\eta, x_{d}, \tau\right)\right\rangle d x_{d}\right) .
\end{array}
$$


Now using $(K 1)$ and $(K 2)$ gives

$$
\begin{array}{r}
|U(\eta, 0, \tau)|^{2}+\alpha \int_{\mathbb{R}^{+}} \operatorname{Re}(\tau) \frac{\left|U\left(\eta, x_{d}, \tau\right)\right|^{2}}{\lambda_{p}(\tau, \eta)^{p-1}} d x_{d} \\
\leq C|F U(\eta, 0, \tau)|^{2} d+\int_{\mathbb{R}^{+}}|f|\left|U\left(\eta, x_{d}, \tau\right)\right| d \delta d \eta d x_{d} .
\end{array}
$$

For the last term on the right hand side, we then use the Cauchy-Schwarz inequality and Young's inequality $a b \leq r a^{2} / 2+b^{2} /(2 r)$ :

$$
\begin{aligned}
|U(\eta, 0, \tau)|^{2} d+\frac{\alpha}{2} \int_{\mathbb{R}^{+}} \operatorname{Re}(\tau) \frac{\left|U\left(\eta, x_{d}, \tau\right)\right|^{2}}{\lambda_{p}(\tau, \eta)^{p-1}} d x_{d} & \leq C|F U(\eta, 0, \tau)|^{2} \\
& +\int_{\mathbb{R}^{+}} \lambda_{p}(\tau, \eta)^{p-1}|f|^{2} d x_{d} .
\end{aligned}
$$

Finally, multiplying the inequality above by $\lambda_{p}^{p-1}$, integrating in $(\operatorname{Im}(\tau), \eta)$ and using the Plancherel theorem we obtain formally $(E D)$. The estimate $(E D s)$ follows from similar arguments that we do not detail. All the calculus above are rigorous if $\widetilde{u} \in \mathcal{S}_{\gamma}$, and in particular it proves $(E D s)$.

The derivation of $(E D)$ in the general case is much more delicate. In fact, even the meaning of $\left.F_{\gamma} \widetilde{u}\right|_{x_{d}=0}$ is not clear if $\widetilde{u} \in L_{\gamma}^{2}$. To clarify it, we will use two results that are proved later (Lemma 2):

- Let $V:=\left\{v \in L_{\gamma}^{2}\left(\mathbb{R}^{d-1} \times \mathbb{R}^{+} \times \mathbb{R}_{t}\right): \partial_{x_{d}} v-G_{\gamma} v \in L^{2}\left(\mathbb{R}^{+} ; H_{\gamma, p}^{p-1}\right)\right\}$, normed by $\|v\|_{L_{\gamma}^{2}}+\left\|\left(\partial_{x_{d}}-G_{\gamma}\right) v\right\|_{L^{2}\left(H_{\gamma, p}^{p-1}\right)}$. The space $\mathcal{C}_{c}^{\infty}\left(\mathbb{R}^{d-1} \times \mathbb{R}^{+} \times \mathbb{R}_{t}\right)$ is dense in $V$.

- The operator of trace on $\left\{x_{d}=0\right\}$, defined on $\mathcal{C}_{c}^{\infty}\left(\mathbb{R}^{d-1} \times \mathbb{R}^{+} \times \mathbb{R}_{t}\right)$ admits two continuous extensions

$$
\begin{aligned}
V & \rightarrow H_{\gamma, p}^{-1 / 2}\left(\mathbb{R}^{d-1} \times \mathbb{R}_{t}\right) \\
\text { and } H^{1}\left(\mathbb{R}^{+} ; H_{\gamma, p}^{p-1}\left(\mathbb{R}^{d-1} \times \mathbb{R}_{t}\right)\right) & \rightarrow H_{\gamma, p}^{(p-1) / 2}\left(\mathbb{R}^{d-1} \times \mathbb{R}_{t}\right)
\end{aligned}
$$

If $\widetilde{u} \in H^{1}\left(\mathbb{R}^{+} ; H_{\gamma, p}^{p-1}\left(\mathbb{R}^{d-1} \times \mathbb{R}_{t}\right)\right)$, let $\left(\widetilde{u_{n}}\right) \in \mathcal{C}_{c}^{\infty}$ be a sequence converging to $\widetilde{u}$ in the space $H^{1}\left(\mathbb{R}^{+} ; H_{\gamma, p}^{p-1}\left(\mathbb{R}^{d-1} \times \mathbb{R}_{t}\right)\right)$. By continuity of the trace operator, $\left.\widetilde{u_{n}}\right|_{x_{d}=0} \rightarrow \widetilde{u}$ in $H_{\gamma, p}^{(p-1) / 2}\left(\mathbb{R}^{d-1} \times \mathbb{R}_{t}\right)$. Applying $(E D)$ and passing to the limit, we obtain $(E D)$ for $\widetilde{u}$. Now in the general case $\widetilde{u} \in L_{\gamma, p}^{2}$, we first note that $\left.F_{\gamma} u\right|_{x_{d}=0}=\varphi$ has a sense since $u$ has a trace $H_{\gamma, p}^{-1 / 2}$ on $\left\{x_{d}=0\right\}$. We take $\rho_{n}: \mathbb{R}^{d-1} \times \mathbb{R}_{t} \rightarrow \mathbb{R}^{+}$a sequence of mollifiers with respect to $\left(x^{\prime}, t\right)$ and consider $\widetilde{u_{n}}:=\rho_{n} * \widetilde{u}$. Since $\widetilde{u_{n}}$ is smooth in $\left(x^{\prime}, t\right)$, we have

$$
\partial_{x} u_{n}=G_{\gamma} u_{n}+\rho_{n} * f^{\prime} \in L^{2}\left(\mathbb{R}^{+} ; H_{\gamma, p}^{p-1}\left(\mathbb{R}^{d-1} \times \mathbb{R}_{t}\right)\right),
$$


and thus $u_{n} \in H^{1}\left(\mathbb{R}^{+} ; H_{\gamma, p}^{p-1}\left(\mathbb{R}^{d-1} \times \mathbb{R}_{t}\right)\right)$. We then use

$$
\begin{gathered}
\rho_{n} * f^{\prime} \rightarrow f^{\prime} \text { in } L^{2}\left(\mathbb{R}^{+} ; H_{\gamma, p}^{p-1}\left(\mathbb{R}^{d-1} \times \mathbb{R}_{t}\right)\right), \\
\rho_{n} * \varphi \rightarrow \varphi \text { in } H_{\gamma, p}^{(p-1) / 2}\left(\mathbb{R}^{d-1} \times \mathbb{R}_{t}\right) .
\end{gathered}
$$

The application of $(E D)$ to $\widetilde{u_{n}}-\widetilde{u_{m}}$ proves that $\left.\widetilde{u_{n}}\right|_{x_{d}=0}$ is a Cauchy sequence in the the Banach space $H_{\gamma, p}^{(p-1) / 2}\left(\mathbb{R}^{d-1} \times \mathbb{R}_{t}\right)$, and thus is convergent. Since the trace $\left.\widetilde{u}\right|_{x_{d}=0}$ exists, by uniqueness of the limit we obtain $\left.u\right|_{x_{d}=0} \in H_{\gamma, p}^{(p-1) / 2}\left(\mathbb{R}^{d-1} \times \mathbb{R}_{t}\right)$, and $\left.\left.\widetilde{u_{n}}\right|_{x_{d}=0} \rightarrow \widetilde{u}\right|_{x_{d}=0}$ in $H_{\gamma, p}^{(p-1) / 2}\left(\mathbb{R}^{d-1} \times \mathbb{R}_{t}\right)$. The inequality $(E D)$ for $u$ follows then from applying it to $\widetilde{u_{n}}$ and passing to the limit in $n$.

The proofs of $(E D e l l)$ and $(E D e l l, s)$ are similar, and we do not detail them.

Lemma 2. The space $\mathcal{C}_{c}^{\infty}\left(\mathbb{R}^{d-1} \times \mathbb{R}^{+} \times \mathbb{R}_{t}\right)$ is dense in $V:=\left\{v \in L_{\gamma}^{2}\left(\mathbb{R}^{d-1} \times \mathbb{R}^{+} \times \mathbb{R}_{t}\right)\right.$ : $\left.\partial_{x_{d}} v-G_{\gamma} v \in L^{2}\left(\mathbb{R}^{+} ; H_{\gamma, p}^{p-1}\right)\right\}$ for the norm $\|v\|_{V}:=\|v\|_{L_{\gamma}^{2}}+\left\|\left(\partial_{x_{d}}-G_{\gamma}\right) v\right\|_{L^{2}\left(H_{\gamma, p}^{p-1}\right)}$.

The trace operator

$$
\begin{array}{r}
\mathcal{C}_{c}^{\infty}\left(\mathbb{R}^{d-1} \times \mathbb{R}^{+} \times \mathbb{R}_{t}\right) \rightarrow \mathcal{C}_{c}^{\infty}\left(\mathbb{R}^{d-1} \times \mathbb{R}_{t}\right), \\
\left.u \in \mathcal{C}_{c}^{\infty}\left(\mathbb{R}^{d-1} \times \mathbb{R}^{+} \times \mathbb{R}_{t}\right) \rightarrow u\right|_{x_{d}=0},
\end{array}
$$

extends continuously

$$
\begin{aligned}
& \text { for } s \in \mathbb{R}^{+}, H^{1}\left(\mathbb{R}^{+} ; H_{\gamma, p}^{s}\left(\mathbb{R}^{d-1} \times \mathbb{R}_{t}\right)\right) \rightarrow H_{\gamma, p}^{s / 2}\left(\mathbb{R}^{d-1} \times \mathbb{R}_{t}\right) \text {, } \\
& \text { and } V \rightarrow H_{\gamma, p}^{-1 / 2}\left(\mathbb{R}^{d-1} \times \mathbb{R}_{t}\right) \text {. }
\end{aligned}
$$

Proof. Let $v \in V$, we denote $\underline{v}$ its extension by 0 for $x_{d}<0$. Multiplying $\underline{v}$ by $\chi \underline{v}$ for $\chi$ a truncature function, one may check that compactly supported functions are dense in $V$. Thus we assume that $\underline{v}$ has compact support. We take a mollifying sequence of the form $\rho_{n} \otimes \psi_{n}$,

$$
\begin{array}{r}
\left.\left.\rho_{n}: \mathbb{R} \longrightarrow \mathbb{R}, \operatorname{supp}(\rho) \subset\right]-1,0\right], \\
\psi_{n}: \mathbb{R}_{t} \times \mathbb{R}^{d-1} \longrightarrow \mathbb{R} .
\end{array}
$$

Let $v_{n}:=\left(\rho_{n} \otimes \psi_{n}\right) * \underline{v}$. For $x_{d}>0$ we have

$$
\begin{aligned}
L \underline{v_{n}}\left(x^{\prime}, x_{d}, t\right) & =L \iint \rho_{n}\left(y_{d}\right) \psi_{n}\left(y^{\prime}, s\right) \underline{v}\left(x^{\prime}-y^{\prime}, x_{d}-y_{d}, t-s\right) d y d s \\
& =L \int_{\mathbb{R}^{d-1} \times \mathbb{R}} \int_{-1 / n}^{0} \rho_{n}\left(y_{d}\right) \psi_{n}\left(y^{\prime}, s\right) v\left(x^{\prime}-y^{\prime}, x_{d}-y_{d}, t-s\right) d y d s \\
& =\int_{\mathbb{R}^{d-1} \times \mathbb{R}} \int_{-1 / n}^{0} \rho_{n}\left(y_{d}\right) \psi_{n}\left(y^{\prime}, s\right) L v\left(x^{\prime}-y^{\prime}, x_{d}-y_{d}, t-s\right) d y_{d} s \\
& =\left(\left(\rho_{n} \otimes \psi_{n}\right) * \underline{L v}\right)\left(x^{\prime}, x_{d}, t\right),
\end{aligned}
$$


(because of the choice $\operatorname{supp}(\rho) \subset]-1,0]$, the region where $L v_{n}$ is 'stiff' lies in $x_{d} \leq 0$ ). Since $\rho_{n} \otimes \psi_{n}$ is a mollifying sequence, this implies

$$
\begin{aligned}
\left.\underline{v_{n}}\right|_{x_{d}>0} & \longrightarrow v\left(L^{2}\left(\mathbb{R}_{t} \times \mathbb{R}^{d-1} \times \mathbb{R}^{+*}\right)\right), \\
\left.\left(L \underline{v_{n}}\right)\right|_{x_{d}>0}=\left.\left(\left(\rho_{n} \otimes \lambda_{n}\right) * \underline{\underline{v}}\right)\right|_{x_{d}>0} & \longrightarrow L v\left(L^{2}\left(\mathbb{R}^{+*} ; H_{\gamma, p}^{p-1}\left(\mathbb{R}_{t} \times \mathbb{R}^{d-1}\right)\right) .\right.
\end{aligned}
$$

Since $v$ is compactly supported so does $v_{n}$ and we conclude that $\mathcal{C}_{c}^{\infty}$ is dense in $V$. We now turn to the trace result. Let $v$ be smoothly defined on $\mathbb{R}^{d-1} \times \mathbb{R}^{+} \times \mathbb{R}_{t}, v_{b}:=$ $\left.v\right|_{x_{d}=0}$, we have

$$
\begin{array}{r}
\left|\widehat{u}_{b}(\tau, \eta)\right|=\left|\int_{\mathbb{R}} \widehat{u}\left(\eta, \eta_{d}, \tau\right) d \eta_{d}\right| \\
\leq \sqrt{\int_{\mathbb{R}}|\widehat{u}|^{2}\left(\gamma^{2 s / p}+\delta^{2 s / p}+|\eta|^{2 s}+\eta_{d}^{2} d \eta_{d}\right.} \sqrt{\int_{\mathbb{R}} \frac{1}{\gamma^{2 s / p}+\delta^{2 s / p}+|\eta|^{2 s}+\eta_{d}^{2}}} d \eta_{d} .
\end{array}
$$

Using the change of variables $\eta_{d}=\widetilde{\eta}_{d} \sqrt{\gamma^{2 s / p}+\delta^{2 s / p}+|\eta|^{2 s}}$ and integrating in $(\tau, \eta)$, we obtain

$$
\begin{aligned}
\left\|u_{b}\right\|_{H_{\gamma, p}^{s / 2}}^{2} & =\int \sqrt{\gamma^{2 s / p}+\delta^{2 s / p}+|\eta|^{2 s}}\left|\widehat{u_{b}}\right|^{2} d \delta d \eta \\
& \leq \pi \int_{\mathbb{R}}|\widehat{u}|^{2}\left(\gamma^{2 s / p}+\delta^{2 s / p}+|\eta|^{2 s}+\eta_{d}^{2}\right) d \eta_{d} d \eta d \delta \\
& \leq \pi\|u\|_{H^{1}\left(\mathbb{R} ; H_{\gamma, p}^{s}\right)} .
\end{aligned}
$$

This inequality allow us to define the trace on $\left\{x_{d}=0\right\}$ for functions in the space $H^{1}\left(\mathbb{R} ; H_{\gamma, p}^{s}\right)$, and then for $v \in H^{1}\left(\mathbb{R}^{+} ; H_{\gamma, p}^{s}\right)$ by taking the trace of an extension $v^{\prime} \in$ $H^{1}\left(\mathbb{R} ; H_{\gamma, p}^{s}\right)$ of $v$.

(Note that the trace does not depend on the chosen extension since it is the limit of traces on $\left\{x_{d}=y\right\}$ for $y \rightarrow 0^{+}$.)

The second trace result can be proved by using the same kind of arguments and the 'jump formula' for distributions $\partial_{x_{d}} \underline{\psi}=\partial_{x_{d}} \psi+\left.\psi\right|_{x_{d}=0} \otimes \delta\left(x_{d}\right)$, see for example [6] Prop. 4.6.I I for a control of the second term in the (not really simpler) case of isotropic Sobolev spaces.

\section{Well-posedness of the BVP}

In this section, we prove Theorem 1, that is, the existence and uniqueness of a solution to $(2)$

$$
\left\{\begin{array}{l}
P u=f,\left(x^{\prime}, x_{d}, t\right) \in \mathbb{R}^{d-1} \times \mathbb{R}^{+} \times \mathbb{R}_{t}, \\
\left.B\left(\partial_{t}, \partial_{x}\right) u\right|_{x_{d}=0}=\varphi
\end{array}\right.
$$


under the hypotheses that $P$ is a strictly dispersive non-characteristic operator, and $B$ satisfies the uniform Kreiss-Lopatinskil condition. The argument of the proof is based on the existence of a dual problem for which we shall only sketch the construction. Indeed the study of hyperbolic boundary value problems relies on the very similar construction of a dual problem, the reader may find it detailed in $[1]$ (§4.4 p.114).

The dual problem We denote by $\langle\cdot, \cdot\rangle$ the scalar product on $L^{2}\left(\mathbb{R}^{d-1} \times \mathbb{R}^{+} \times \mathbb{R}_{t}\right)$, and $(\cdot, \cdot)$ the scalar product on $L^{2}\left(\mathbb{R}^{d-1} \times \mathbb{R}_{t}\right)$. The product $\langle u, v\rangle$ will often be considered as a dual product $\left\langle e^{-\gamma t} u, e^{\gamma t} v\right\rangle$ in Sobolev spaces without saying it explicitly.

Proposition 7. If $A^{\gamma}$ is a tangential Fourier multiplier with parameter, we denote ${ }_{\gamma} A:=e^{-\gamma t} A^{\gamma} e^{\gamma t}$. Let $\Gamma$ be a positive large integer.

There exists smooth matricial symbols $\left(F^{\prime}, m, n\right)(\widehat{\tau}, \widehat{\eta}, \varepsilon)$ defined on $\mathbb{S}_{p}^{+} \times[0,1 / \Gamma]$ and corresponding operators ${ }_{\gamma} F^{\prime},{ }_{\gamma} M,{ }_{\gamma} N$ of order 0 such that if $\widetilde{u}$ is a solution of

$$
\left\{\begin{array}{l}
L \widetilde{u}:=\partial_{x_{d}} \widetilde{u}-G_{\gamma} \widetilde{u}=f^{\prime} \\
\left.F_{\gamma} \widetilde{u}\right|_{x_{d}=0}=\varphi
\end{array}\right.
$$

then we have the (formal) identity

$$
\langle L \widetilde{u}, \widetilde{v}\rangle=\left\langle\widetilde{u}, L^{\prime} \widetilde{v}\right\rangle+\left(F_{\gamma} \widetilde{u}(0),{ }_{\gamma} M \widetilde{v}(0)\right)+\left(N_{\gamma} \widetilde{u}(0),{ }_{\gamma} F^{\prime} \widetilde{v}(0)\right),
$$

with $L^{\prime}:=-\partial_{x_{d}}-{ }_{\gamma} G^{*}$. Moreover, $m$ is an isomorphism $\operatorname{Ker}\left(F^{\prime}\right) \rightarrow \operatorname{Im}(m)$ for $(\widehat{\tau}, \widehat{\eta}, \varepsilon) \in$ $\mathbb{S}_{p}^{+} \times[0,1 / \Gamma]$.

We call dual problem the system

$$
\left\{\begin{array}{l}
L^{\prime} \widetilde{v}=f_{1}^{\prime}, x_{d} \geq 0, t \in \mathbb{R} \\
\left.{ }_{\gamma} F^{\prime} \widetilde{v}\right|_{x_{d}=0}=\varphi_{1}, t \in \mathbb{R},
\end{array}\right.
$$

and define the backward in time Kreiss-Lopatinskiu condition exactly as the KreissLopatinski condition but for operators of the form ${ }_{\gamma} A$ instead of $A_{\gamma}$. The boundary operator ${ }_{-\gamma} F^{\prime}$ satisfies backward (KLU) if and only if $F_{\gamma}$ satisfies (KLU). If backward $(K L U)$ is satisfied, we have the backward analogous of $(E D)$ : for $\widetilde{v}$ solution of $(20)$ and $\gamma \geq \Gamma$ large enough

$$
\begin{gathered}
\gamma\|\widetilde{v}\|_{L_{-\gamma}^{2}\left(\mathbb{R}^{d-1} \times \mathbb{R}^{+} \times \mathbb{R}_{t}\right)}^{2}+|\widetilde{v}(0)|_{H_{-\gamma, p}^{(p-1) / 2}\left(\mathbb{R}^{d-1} \times \mathbb{R}_{t}\right)}^{2} \\
\lesssim \frac{1}{\gamma}\left\|L^{\prime} \widetilde{v}\right\|_{L^{2}\left(\mathbb{R}^{+} ; H_{-\gamma, p}^{p-1}\left(\mathbb{R}^{d-1} \times \mathbb{R}_{t}\right)\right)}^{2}+\left.\left.\right|_{-\gamma} F^{\prime} \widetilde{v}(0)\right|_{H_{-\gamma, p}^{(p-1) / 2}\left(\mathbb{R}^{d-1} \times \mathbb{R}_{t}\right)} ^{2}
\end{gathered}
$$

and the backwards analogous of $(E D s)$

$$
\begin{aligned}
& \forall \gamma \geq \Gamma, \gamma\|\widetilde{v}\|_{L^{2}\left(\mathbb{R}^{+} ; H_{-\gamma, p}^{s}\right)}^{2}+|\widetilde{v}(0)|_{L^{2}\left(\mathbb{R}^{+} ; H_{-\gamma, p}^{s+(p-1) / 2}\right)}^{2} \\
& \lesssim \frac{1}{\gamma}\left\|L^{\prime} \widetilde{v}\right\|_{L^{2}\left(\mathbb{R}^{+} ; H_{-\gamma, p}^{s+p-1}\right)}^{2}+\left|{ }_{\gamma} F^{\prime} \widetilde{v}(0)\right|_{L^{2}\left(\mathbb{R}^{+} ; H_{-\gamma, p}^{s+(p-1) / 2}\right)}^{2}
\end{aligned}
$$

stands too. 
Sketch of proof. Since $\langle L \widetilde{u}, \widetilde{v}\rangle=\left\langle\widetilde{u}, L^{*} \widetilde{v}\right\rangle-\left.\left.\int_{\mathbb{R}^{d-1} \times \mathbb{R}_{t}} \widetilde{u}\right|_{x_{d}=0} \overline{\widetilde{v}}\right|_{x_{d}=0}$, it suffices to find symbols $F^{\prime}, m, n$ such that, if we still denote $F$ the symbol of $F_{\gamma}$

$$
m^{*} F+\left(F^{\prime}\right)^{*} n=-I_{m n p} .
$$

Since $F$ has a constant rank, there exists a smooth matrix $n$ such that $\left(\begin{array}{l}F \\ n\end{array}\right) \in G L_{m p n}$ (this comes from the fact that the set $\mathbb{S}_{p}^{+} \times[0,1 / C]$ is contractible, and thus the vector bundle $E^{-}$is trivial). Let $\left(\begin{array}{c}F \\ n\end{array}\right)^{-1}:=\left(m^{*}\left(F^{\prime}\right)^{*}\right)$, where $F^{\prime}$ has $m n p-\operatorname{rank}(F)$ lines. It is easily checked that for this choice of $m, n, F^{\prime}$ we have $(21)$, and moreover $\operatorname{Ker}\left(F^{\prime}\right)=$ $\operatorname{Ker}(F)^{\perp}$. The backward Kreiss-Lopatinskiı̌ condition amounts to

$$
E^{-}\left(-g^{*}\right) \cap \operatorname{Ker}\left(F^{\prime}\right)=\{0\},
$$

and the equivalence with the Kreiss-Lopatinskil condition is in fact a consequence of the -not completely straightforward- formula (again, see [1] §4.4 p.114 for details)

$$
\left(E^{-}\left(-g^{*}\right)+\operatorname{Ker}\left(F^{\prime}\right)\right)^{\perp}=E^{-}(g) \cap \operatorname{Ker}(F) .
$$

Using Proposition 4, we obtain similarly the equivalence between KLU for $F$ and backward KLU for $F^{\prime}$.

We show now how the existence of a solution to (12) can be deduced from the proposition above. Let $E:=\left\{\psi \in \mathcal{S}_{-\gamma}:\left.{ }_{\gamma} F^{\prime} \psi\right|_{x_{d}=0}=0\right\}$. We define the linear form

$$
\begin{aligned}
T: L^{\prime}(E) & \rightarrow \mathbb{C}, \\
L^{\prime}(\psi) & \rightarrow\langle f, \psi\rangle-\left(\varphi,\left.{ }_{\gamma} M \psi\right|_{x_{d}=0}\right) .
\end{aligned}
$$

We use the condensed notation $\|\theta\|_{s, \gamma, p}:=\|\theta\|_{L^{2}\left(\mathbb{R}^{+} ; H_{\gamma, p}^{s}\left(\mathbb{R}^{d-1} \times \mathbb{R}_{t}\right)\right)}$. Using the identification $\left(H_{\gamma, p}^{s}\left(\mathbb{R}^{d-1} \times \mathbb{R}_{t}\right)\right)^{\prime}=H_{-\gamma, p}^{-s}\left(\mathbb{R}^{d-1} \times \mathbb{R}_{t}\right)$ and the a priori estimate $(b E D s)$ we find

$$
\begin{aligned}
T\left(L^{\prime} \psi\right)= & \leq\|f\|_{p-1, \gamma, p}\|\psi\|_{1-p,-\gamma, p}+|\varphi|_{\frac{p-1}{2}, \gamma, p}|\psi|_{\frac{1-p}{2},-\gamma, p} \\
& \lesssim\left\|L^{\prime} \psi\right\|_{L_{-\gamma}^{2}}\left(\frac{1}{\gamma}\|f\|_{p-1, \gamma, p}+\frac{1}{\sqrt{\gamma}}|\varphi|_{\frac{p-1}{2}, \gamma, p}\right) .
\end{aligned}
$$

Thus $T$ is continuous for the $L_{-\gamma}^{2}$ norm. Hahn-Banach's theorem and the Riesz's representation theorem give the existence of $\widetilde{u} \in L_{\gamma}^{2}$ such that for any $\psi \in E$,

$$
\langle\bar{f}, \psi\rangle-\left(\varphi,\left.{ }_{\gamma} M \psi\right|_{x_{d}=0}\right)=\left\langle u, L^{\prime} \psi\right\rangle .
$$

In particular, if $\psi \in C_{c}^{\infty}\left(\mathbb{R}^{d-1} \times \mathbb{R}^{+*} \times \mathbb{R}_{t}\right)$, we have

$$
\left\langle u, L^{\prime} \psi\right\rangle=\langle f, \psi\rangle
$$


and thus $L \widetilde{u}=f$ in the sense of distribution. According to lemma $2,\left.\widetilde{u}\right|_{x_{d}=0}$ exists and belongs to $H_{\gamma, p}^{-1 / 2}$. Now, since the trace of $\widetilde{u}$ is defined we may use the identity (19):

$$
\begin{array}{r}
\forall \psi \in E,\langle f, \psi\rangle-\left(\varphi,{ }_{\gamma} M \psi(0)\right)=\left\langle\widetilde{u}, L^{\prime} \psi\right\rangle \\
\Rightarrow\left\langle F_{\gamma} \widetilde{u}(0)-\varphi,{ }_{\gamma} M \psi(0)\right\rangle=0 .
\end{array}
$$

It suffices to check that $\left.{ }_{\gamma} M(E)\right|_{x_{d}=0}$ is a space 'large enough' to conclude that $F_{\gamma} \widetilde{u}(0)=$ $\varphi$.

We first note that any function in $\mathcal{C}_{c}^{\infty}\left(\mathbb{R}^{d-1} \times \mathbb{R}_{t}\right)$ is the trace of a function in $\mathcal{C}_{c}^{\infty}\left(\mathbb{R}^{d-1} \times\right.$ $\mathbb{R}^{+} \times \mathbb{R}_{t}$ ) (this is a consequence of Borel's lemma). As $m(\widehat{\tau}, \widehat{\eta}, \varepsilon)$ is an isomorphism $\operatorname{Ker}\left(F^{\prime}(\widehat{\tau}, \widehat{\eta}, \varepsilon)\right) \rightarrow \operatorname{Im}(M)$ the compacity of $\mathbb{S}_{p}^{+} \times[0,1 / \Gamma]$ implies that $m^{-1}$ is uniformly bounded. If $\psi \in \mathcal{C}_{c}^{\infty}\left(\mathbb{R}^{d-1} \times \mathbb{R}^{+} \times \mathbb{R}_{t}\right.$, the boundedness of $m^{-1}$ implies that the inverse Fourier-Laplace transform of $m^{-1} \widehat{\psi}\left(-\gamma+i \delta, \eta, x_{d}\right)$ belongs to $\mathcal{S}_{-\gamma}\left(\mathbb{R}^{d-1} \times \mathbb{R}^{+} \times \mathbb{R}_{t}\right)$ and therefore $\left.{ }_{\gamma} M(E)\right|_{x_{d}=0} \supset \mathcal{C}_{c}^{\infty}\left(\mathbb{R}^{d-1} \times \mathbb{R}^{+} \times \mathbb{R}_{t}\right)$. Finally by density of $\mathcal{C}_{c}^{\infty}$ in $H_{\gamma, p}^{(p-1) / 2}$ we get $F_{\gamma} \widetilde{u}=\varphi$.

To summarize:

- The problem (2) has been reformulated as (12),

- The existence of a solution for (12) is obtained thanks to Theorem 3 and the construction of a dual problem,

- The uniqueness follows from theorem 3.

These three points imply the well posedness of the boundary value problems (12) and (2). Now we recall that $\widetilde{u}=\left(\begin{array}{c}\Lambda_{\gamma}^{m p-1} u \\ \vdots \\ \partial_{x_{d}}^{m p-1} u\end{array}\right)$. In particular,

$$
\|\widetilde{u}\|_{L_{\gamma}^{2}}=\|u\|_{H_{\gamma, p}^{m p-1}},|\widetilde{u}(0)|_{H_{\gamma, p}^{(p-1) / 2}}=\sum_{j=0}^{m p-1}\left|\partial_{x_{d}}^{j} u(0)\right|_{H_{\gamma, p}^{m p-1-j+(p-1) / 2}},
$$

and we see that the transcription of $(E D)$ (p.14) for $u$ is precisely (3), this ends the proof of theorem 1.

In the special case of a symbol of elliptic spatial part, we introduce the spaces

$$
\forall(s, r) \in \mathbb{R}^{+}, H_{\gamma, p, t}^{s, r}\left(\mathbb{R}^{d} \times \mathbb{R}_{t}\right):=\left\{u \in L_{\gamma}^{2}: \iint\left(|\tau|^{2 r / p}+|\eta|^{2 s}\right)|\widehat{u}|^{2} d \delta d \eta<\infty\right\},
$$

and $H^{s, r}\left(\Omega \times \mathbb{R}_{t}\right)$ is defined by restriction on $\Omega \times \mathbb{R}_{t}$ of functions in $H_{\gamma, p, t}^{s, r}\left(\mathbb{R}^{d} \times \mathbb{R}_{t}\right)$. By applying the same proof and the a priori estimates (EDell) and (EDell,s) (p.14), we obtain the following result. 
Corollary 1. Under the same hypotheses as in Theorem 1, if moreover $P$ has an elliptic spatial part (see (18) then the problem (2) has an unique solution $u \in H_{\gamma, p}^{m p-1}$, and it satisfies the estimate

$$
\begin{aligned}
\gamma\|u\|_{H_{\gamma, p}^{m p-1}} & +\sum_{j=0}^{m p-1}\left|\partial_{x_{d}}^{j} u\right|_{H_{\gamma, p}^{m p-1-j, m p-1-j+(p-1) / 2}} \\
& \leq C\left(\frac{\|f\|_{L^{2}\left(\mathbb{R}^{d-1} \times \mathbb{R}^{+} ; H_{\gamma}^{(p-1) / p}\right)}}{\gamma}+|\varphi|_{H_{\gamma, p}^{(0, p-1) / 2}}\right) .
\end{aligned}
$$

Well-posedness with more regularity For $\xi \in \mathbb{R}^{d}$ (dual variable of $x$ ) we denote $\xi=\left(\xi^{\prime}, \xi_{d}\right)$. We define the Sobolev spaces

$$
\begin{aligned}
& \forall(s, r) \in \mathbb{R}^{+}, H_{\gamma, p, x^{\prime}, t}^{s, r}\left(\mathbb{R}^{d} \times \mathbb{R}_{t}\right):=\left\{u \in L_{\gamma}^{2}:\right. \\
& \left.\iint\left(\left(|\tau|^{2 / p}+\left|\xi^{\prime}\right|^{2}\right)^{r}+\left|\xi_{d}\right|^{2 s}\right)|\widehat{u}|^{2} d \delta d \eta<\infty\right\}
\end{aligned}
$$

the spaces $H_{\gamma, p, x^{\prime}, t}^{s, r}\left(\Omega \times \mathbb{R}_{t}\right)$ are defined by restriction.

Corollary 2. If the data $(\varphi, f)$ belong to $H_{\gamma, p}^{r+(p-1) / 2}\left(\mathbb{R}^{d-1} \times \mathbb{R}_{t}\right) \times H_{\gamma, p, x^{\prime}, t}^{r, r+p-1}\left(\mathbb{R}^{d-1} \times \mathbb{R}^{+} \times \mathbb{R}_{t}\right)$ for $r \geq 1$ integer, then the solution of (2) belongs to $H_{\gamma, p}^{m p-1+r}$.

In particular, if the data are in $\cap_{r \geq 0}\left(H_{\gamma, p}^{r+(p-1) / 2}\left(\mathbb{R}^{d-1} \times \mathbb{R}_{t}\right) \times H_{\gamma, p, x^{\prime}, t}^{r, r+p-1}\left(\mathbb{R}^{d-1} \times \mathbb{R}^{+} \times \mathbb{R}_{t}\right)\right)$, the solution is $\mathcal{C}^{\infty}$.

Proof. We only show how to derive a priori estimates for smooth functions, the actual proof being analogous, by density arguments, to the one for $(E D)$. If $r=1$, we use the reformulation (12). If $\widetilde{u} \in \mathcal{S}_{\gamma}$ the estimate $(E D s)$ (p.14) implies

$$
\forall \gamma \geq \Gamma,\|\widetilde{u}\|_{L^{2}\left(\mathbb{R}^{+} ; H_{\gamma, p}^{1}\right)}^{2} \lesssim\left\|f^{\prime}\right\|_{L^{2}\left(\mathbb{R}^{+} ; H_{\gamma, p}^{1+p-1}\right)}^{2}+|\varphi|_{H_{\gamma, p}^{1+(p-1) / 2}}^{2}
$$

Since $G_{\gamma}$ acts on the tangential variables $\left(x^{\prime}, t\right)$ and is of order 1 , this implies

$$
\left\|G_{\gamma} \widetilde{u}\right\|_{L_{\gamma}^{2}} \lesssim\left\|f^{\prime}\right\|_{L^{2}\left(\mathbb{R}^{+} ; H_{\gamma, p}^{1+p-1}\right)}+|\varphi|_{H_{\gamma, p}^{1+(p-1) / 2}}
$$

then we use $\partial_{x_{d}} \widetilde{u}-G_{\gamma} u=f^{\prime}$ with $f^{\prime}=\left(\begin{array}{c}0 \\ \vdots \\ f\end{array}\right)$ to deduce

$$
\left\|\partial_{x_{d}} \widetilde{u}\right\|_{L_{\gamma}^{2}} \lesssim\|f\|_{L^{2}\left(\mathbb{R}^{+} ; H_{\gamma, p}^{1+p-1}\right)}^{2}+|\varphi|_{H_{\gamma, p}^{1+(p-1) / 2},}
$$


(22) and (23) mean for $u$

$$
\|u\|_{H_{\gamma, p}^{m p-1+1}} \lesssim\|f\|_{L^{2}\left(\mathbb{R}^{+} ; H_{\gamma, p}^{1+p-1}\right)}+|\varphi|_{H_{\gamma, p}^{1+(p-1) / 2}}
$$

If $r=2$, the same argument as above implies the analogue of (22)

$$
\forall \gamma \geq \Gamma,\|\widetilde{u}\|_{L^{2}\left(\mathbb{R}^{+} ; H_{\gamma, p}^{2}\right)}^{2} \lesssim\left\|f^{\prime}\right\|_{L^{2}\left(\mathbb{R}^{+} ; H_{\gamma, p}^{2+p-1}\right)}+|\varphi|_{H_{\gamma, p}^{2+(p-1) / 2},}
$$

and the analogous of $(23)$ for $\partial_{x_{d}} \widetilde{u}$. Then derivating $\partial_{x_{d}} \widetilde{u}-G_{\gamma} \widetilde{u}=f^{\prime}$ with respect to $x_{d}$ gives

$$
\left\|\partial_{x_{d}}^{2} \widetilde{u}\right\|_{L_{\gamma}^{2}}=\left\|G_{\gamma} \partial_{x_{d}} \widetilde{u}+\partial_{x_{d}} f^{\prime}\right\|_{L_{\gamma}^{2}} \lesssim\left\|\partial_{x_{d}} f^{\prime}\right\|_{L^{2}\left(\mathbb{R}^{+} ; H_{\gamma, p}^{2+p-1}\right)}+|\varphi|_{H_{\gamma, p}^{2+(p-1) / 2}}
$$

the transcription of these estimates for $u$ gives

$$
\|\widetilde{u}\|_{H_{\gamma, p}^{2+m p-1}} \lesssim\|f\|_{H_{\gamma, p, x^{\prime}, t}^{1,2+p-1}}+|\varphi|_{H_{\gamma, p}^{2+(p-1) / 2}} .
$$

The proof of similar estimates for $r \geq 3$ follows by recurrence.

\section{The initial boundary value problem}

This section is divided in two paragraphs. In the first one, we prove that the boundary value problem with zero initial data is well posed. This is merely a consequence of the well-posedness for the pure boundary value problem and some technical lemmas. Secondly we turn to the harder problem of incorporating non-zero initial data: for this case we only give a result for the linear Schrödinger equation, although we believe that our method of proof is general. This is in fact an adaptation of the method used by Rauch [21] to prove pointwise time estimates for the solutions of strictly hyperbolic boundary value problems.

In order to avoid excessive technicity we assume here that $B$ is a constant matrix, but the results may be extended (up to using a quasi-homogeneous pseudo-differential calculus with parameter) to the case where $B=B_{\gamma}$ is a tangential Fourier-Laplace multiplier of $p$-order 0 (this assumption is only used for Proposition 8).

Zero initial data Our aim here is to prove the well posedness of

$$
\left\{\begin{array}{l}
P u=f \\
\left.B_{\gamma}\left(\partial_{t}, \partial_{x}\right) u\right|_{x_{d}=0}=\varphi \\
\left.u\right|_{t=0}=0
\end{array}\right.
$$


Lemma 3. For $k \geq 0$, if $\psi \in H_{\gamma, p}^{k}\left(\mathbb{R}^{d-1} \times \mathbb{R}^{+} \times \mathbb{R}_{t}\right)$ and $\theta: \mathbb{R}_{t} \rightarrow \mathbb{R}$ is a smooth bounded function as well as its derivatives, then the mapping $(x, t) \rightarrow \theta(t) \psi(x, t)$ is in $H_{\gamma, p}^{k}$, and we have $\|\theta \psi\|_{H_{\gamma, p}^{k}} \leq C\|\psi\|_{H_{\gamma, p}^{k}}$, with $C$ only depending on the $L^{\infty}$ norm of the derivatives of $\varphi$ of order $\leq[k / p]+1$ (where $[\cdot]$ denotes the integer part).

Proof. If $k$ is a multiple of $p$, we have for $u \in H_{\gamma, p}^{k}$

$$
e^{-\gamma t \partial_{t}^{k / p}} u\left(\eta, x_{d}, i \delta\right)=(\gamma+i \delta)^{k / p} \widehat{u}\left(\eta, x_{d}, \gamma+i \delta\right) .
$$

Therefore it suffices to replace $u$ by $\theta \psi$ in the equation above and apply Leibniz's formula. The general case follows by interpolation.

Proposition 8. If $B_{\gamma}$ is a constant matrix, there exists $\Gamma>0$ such that for any $\gamma \geq \Gamma$ the solution of (2) does not depend on $\gamma$.

Proof. Let $\gamma_{1}<\gamma_{2}$ be real positive numbers, and $u_{1} \in H_{\gamma_{1}, p}^{m p-1}$ and $u_{2} \in H_{\gamma_{2}, p}^{m p-1}$ be the corresponding solutions of the boundary value problem. We first assume that $\gamma_{2}-\gamma_{1} \leq 1$. Let $\theta: \mathbb{R}_{t} \rightarrow \mathbb{R}^{+*}$ be a smooth function such that $\theta(t)=1$ for $t \leq-1, \theta(t)=e^{\left(\gamma_{1}-\gamma_{2}\right) t}$ for $t \geq 0$. Obviously $\theta u_{2} \in H_{\gamma_{1}, p}^{m p-1}$, and $\theta u_{1}$ is still in $H_{\gamma_{1}, p}^{m p-1}$ by Lemma 3. Since $P u_{1}=f$ and $P u_{2}=f$ we have

$$
\theta P\left(\partial_{t}, D_{x}\right)\left(u_{2}-u_{1}\right)=0 \Rightarrow P\left(\partial_{t}, D_{x}\right)\left(\theta\left(u_{2}-u_{1}\right)\right)+[\theta, P]\left(\frac{1}{\theta}\left(\theta\left(u_{2}-u_{1}\right)\right)\right)=0 .
$$

Since $P$ is of $p$-order $m p$ and $\theta$ only depends on $t$, the commutator $[\theta, P]$ is of $p$-order $p(m-1)$, and Lemma 3 implies that the operator $v \rightarrow[\theta, P]\left(\frac{1}{\theta} v\right)$ is continuous $H_{\gamma, p}^{k} \rightarrow$ $H_{\gamma, p}^{k-p(m-1)}\left(\mathbb{R}^{d-1} \times \mathbb{R}^{+} \times \mathbb{R}_{t}\right)$, with a norm independant of $\gamma_{1}, \gamma_{2}$ because of the choice $\left|\gamma_{1}-\gamma_{2}\right| \leq 1$. If $B_{\gamma}$ is a constant matrix, $[\theta, B]=0$ thus $B\left(\theta\left(u_{2}-u_{1}\right)\right)=0$ (this is the only point of having $B_{\gamma}$ constant, in the general case, this would become a commutator estimate). We now apply the a priori estimate (3) by considering $[\theta, P]\left(\frac{1}{\theta}\left(\theta\left(u_{2}-u_{1}\right)\right)\right)$ as a forcing term to obtain

$$
\begin{aligned}
\gamma_{1}\left\|\theta\left(u_{2}-u_{1}\right)\right\|_{H_{\gamma_{1}, p}^{m p-1}}^{2} & +\sum_{j=0}^{m p-1}\left|\theta \partial_{x_{d}}^{j}\left(u_{2}-u_{1}\right)\right|_{H_{\gamma_{1}, p}^{m p-1-j+(p-1) / 2}}^{2} \\
& \leq \frac{C}{\gamma_{1}}\left\|[\theta, P]\left(\frac{1}{\theta}\left(\theta\left(u_{2}-u_{1}\right)\right)\right)\right\|_{L^{2}\left(H_{\gamma_{1}, p}^{p-1}\right)}^{2} \\
& \leq \frac{C^{\prime}}{\gamma_{1}}\left\|\theta\left(u_{2}-u_{1}\right)\right\|_{\left.H_{\gamma_{1}, p}^{m p-1}\right)}^{2} .
\end{aligned}
$$

Now if $\Gamma$ is large enough and $\gamma_{1}+1 \geq \gamma_{2} \geq \gamma_{1} \geq \Gamma$, the inequality above implies $\theta\left(u_{2}-u_{1}\right)=0$ so $u_{2}=u_{1}$ because $\theta$ does not cancel. The result for general $\gamma_{1}, \gamma_{2}$ greater than $\Gamma$ follows by connectedness of $\mathbb{R}$. 
Lemma 4. Let $u$ be the solution of

$$
\left\{\begin{array}{l}
P u=f \\
\left.B_{\gamma} u\right|_{x_{d}=0}=\varphi
\end{array}\right.
$$

If for $t \leq 0, f^{\prime}=0$ and $\varphi=0$, then $\widetilde{u}=0$ for $t<0$.

Proof. According to Proposition 8, there exists $\Gamma>0$ such that for $\gamma \geq \Gamma u$ does not depend on $\gamma$. Given any $\varepsilon>0$

$$
\|u\|_{\left.\left.L_{\Gamma}^{2}\left(\mathbb{R}^{d-1} \times \mathbb{R}^{+} \times\right]-\infty,-\varepsilon\right]\right)} \leq e^{-(\gamma-\Gamma) \varepsilon}\|u\|_{\left.\left.L_{\gamma}^{2}\left(\mathbb{R}^{d-1} \times \mathbb{R}^{+} \times\right]-\infty,-\varepsilon\right]\right)} .
$$

The a priori estimate (3) implies

$$
e^{(\gamma-\Gamma) \varepsilon}\|u\|_{\left.\left.L_{\Gamma}^{2}\left(\mathbb{R}^{d-1} \times \mathbb{R}^{+} \times\right]-\infty,-\varepsilon\right]\right)} \lesssim \frac{1}{\gamma}\|f\|_{L^{2} H_{\gamma, p}^{p-1}}+\|\varphi\|_{H_{\gamma, p}^{(p-1) / 2}},
$$

and we see that if for any $\varepsilon>0$

$$
\lim _{\gamma \rightarrow \infty} e^{-(\gamma-\Gamma) \varepsilon}\left(\frac{1}{\gamma}\|f\|_{L^{2} H_{\gamma, p}^{p-1}}+\|\varphi\|_{H_{\gamma, p}^{(p-1) / 2}}\right)=0
$$

then $u=0$ for $t \leq-\varepsilon$ arbitrarily in $\varepsilon$, which is the required result.

Let $\theta: \mathbb{R} \rightarrow \mathbb{R}^{+}$be such that $\theta(t)=e^{(\Gamma-\gamma) t}$ for $t \geq 0, \theta(t)=0$ for $t \leq-1$. The hypothesis of support for $f$ and $\varphi$ implies $e^{(\Gamma-\gamma) t} f=\theta f$ and $e^{(\Gamma-\gamma t)} \varphi=\theta \varphi$. Moreover by definition of the spaces $H_{\gamma, p}^{s},\|\cdot\|_{H_{\gamma, p}^{s}} \leq(\gamma / \Gamma)^{s}\left\|e^{(\Gamma-\gamma) t} \cdot\right\|_{H_{\Gamma, p}^{s}}$, in particular

$$
\|f\|_{H_{\gamma, p}^{p-1}} \leq(\gamma / \Gamma)^{s}\|\theta f\|_{H_{\Gamma, p}^{p-1}}\|\varphi\|_{H_{\gamma, p}^{(p-1) / 2}} \leq(\gamma / \Gamma)^{s}\|\theta \varphi\|_{H_{\Gamma, p}^{(p-1) / 2}}
$$

But $\theta$ is bounded as well as its derivatives at most polynomially in $\gamma$, and finally Lemma 3 implies (25).

If $J$ is an interval, similarly as in section 1 we can define $H_{\gamma, p}^{s}\left(\mathbb{R}^{d-1} \times J\right)$ as the space of restrictions on $\mathbb{R}^{d-1} \times J$ of functions in $H_{\gamma, p}^{s}\left(\mathbb{R}^{d-1} \times \mathbb{R}_{t}\right)$, and $H_{0, \gamma, p}^{s}$ as the closure of $\mathcal{C}_{c}^{\infty}\left(\mathbb{R}^{d-1} \times \mathbb{R}_{t}^{+*}\right.$. We define in the same way for $H_{\gamma, p}^{s}\left(\mathbb{R}^{d-1} \times \mathbb{R}^{+} \times J\right)$ as restrictions on $\mathbb{R}^{d-1} \times \mathbb{R}^{+} \times J$.

Before showing our well-posedness result for the initial boundary value problem, let us explain what is the meaning of ' $\left.\partial_{t}^{m-1} u\right|_{t=0}$ ': by a slight modification of Lemma 2 one may prove that for any $u \in H_{\gamma, p}^{m p-1}\left(\mathbb{R}^{d} \times \mathbb{R}_{t}\right)$ such that $P u=f \in L_{\gamma}^{2},\left(u, \partial_{t} u, \cdots, \partial_{t}^{m-1} u\right)$ admits a trace on $\left\{(x, t) \in \mathbb{R}^{d} \times\{0\}\right\}$, this fact may be localized by using suitable smooth truncature functions, and then by partition of the unity this give a sense to the trace of $\partial_{t}^{m-1} u$ on $\mathbb{R}^{d-1} \times \mathbb{R}^{+*} \times\{0\}$. 
Theorem 4. Under the same hypotheses as in Theorem 1 and the compatibility conditions $\varphi \in H_{0, \gamma, p}^{(p-1) / 2}\left(\mathbb{R}^{d-1} \times \mathbb{R}_{t}^{+}\right), f \in L^{2}\left(\mathbb{R}^{+} ; H_{0, \gamma, p}^{p-1}\left(\mathbb{R}^{d-1} \times \mathbb{R}_{t}^{+}\right)\right)$

$$
\left\{\begin{array}{l}
P u=f, t>0 \\
\left.B_{\gamma} u\right|_{x_{d}=0}=\varphi, t>0 \\
\left.u\right|_{t=0}=\left.\partial_{t} u\right|_{t=0}=\cdots=\left.\partial_{t}^{m-1} u\right|_{t=0}=0
\end{array}\right.
$$

admits an unique solution that satisfies moreover the a priori estimate

$$
\begin{array}{r}
\gamma\|u\|_{H_{\gamma, p}^{m p-1}\left(\mathbb{R}^{d-1} \times \mathbb{R}^{+} \times \mathbb{R}_{t}^{+}\right)}+\sum_{j=0}^{m p-1}\left|\partial_{x_{d}}^{j} u\right|_{H_{\gamma, p}^{m p-1-j+(p-1) / 2}\left(\mathbb{R}^{d-1} \times \mathbb{R}_{t}^{+}\right)} \\
\left.\left.\leq C\left(\frac{1}{\gamma}\|f\|_{L^{2}\left(\mathbb{R}^{+} ; H_{\gamma, p}^{p-1}\right)}\left(\mathbb{R}^{d-1} \times \mathbb{R}_{t}^{+}\right)\right)+|\varphi|_{H_{\gamma, p}^{(p-1) / 2}}\left(\mathbb{R}^{d-1} \times \mathbb{R}_{t}^{+}\right)\right)\right),
\end{array}
$$

Proof. By assumption on $(f, \varphi)$, their extensions $(f, \underline{\varphi})$ by 0 for $t<0$ belong to $L^{2}\left(\mathbb{R}^{+} ; H_{\gamma, p}^{p-1}\left(\mathbb{R}^{d-1} \times\right.\right.$ $\left.\left.\mathbb{R}_{t}\right)\right) \times H_{\gamma, p}^{(p-1) / 2}\left(\mathbb{R}^{d-1} \times \mathbb{R}_{t}\right)$. Let $v$ be the solution of the boundary value problem

$$
\left\{\begin{array}{l}
P v=\underline{f} \\
\left.B_{\gamma} v\right|_{x_{d}=0}=\underline{\varphi}
\end{array}\right.
$$

By Lemma 2, $v=0$ for $t<0$, thus $\partial_{t} v, \cdots, \partial_{t}^{m-1} v$ vanish too for $t<0$. The discussion on traces preceeding the theorem and the jump formula implies then that $\left.\left(v, \partial_{t} v, \cdots, \partial_{t}^{m-1} v\right)\right|_{t=0}=0$. Taking $u=\left.v\right|_{t \geq 0}$ we obtain the existence of a solution.

Now if $u_{1}$ is another solution, the hypothesis $\left.u_{1}\right|_{t=0}=\cdots=\left.\partial_{t}^{m-1} u_{1}\right|_{t=0}=0$ implies that its extension $\underline{u_{1}}$ by 0 for $t<0$ is in $H_{\gamma, p}^{m p-1}\left(\mathbb{R}^{d-1} \times \mathbb{R}^{+} \times \mathbb{R}_{t}\right)$, and is a solution of the boundary value problem (27). The uniqueness part of theorem 1 implies $u_{1}=v$, implying itself $u_{1}=u$.

Corollary 3. 1) In Theorem 1 , the result is still true if we replace $\mathbb{R}_{t}$ by $\left.]-\infty, T\right]$ for any $T \in \mathbb{R}$.

2) In Theorem 4, the result is still true if we replace $[0, \infty[$ by $[0, T]$ for any $T>0$, and assume that $\varphi$, resp. $f$, is the restriction on $\mathbb{R}^{d-1} \times[0, T]$, resp. $\mathbb{R}^{d-1} \times \mathbb{R}^{+} \times[0, T]$ of a function in $H_{0, \gamma, p}^{(p-1) / 2}\left(\mathbb{R}^{d-1} \times \mathbb{R}_{t}^{+}\right)$, resp. $L^{2}\left(\mathbb{R}^{+} ; H_{0, \gamma, p}^{p-1}\left(\mathbb{R}^{d-1} \times \mathbb{R}_{t}^{+}\right)\right)$.

3) The results for higher regularity of Corollary 2 are still true if $\mathbb{R}_{t}$ is replaced by $[0, T]$ or $[0, \infty[$ in the case of zero initial data.

Proof. 1) We have to prove the well posedness of

$$
\left\{\begin{array}{l}
\left.\left.P u=f,(x, t) \in \mathbb{R}^{d-1} \times \mathbb{R}^{+} \times\right]-\infty, T\right] \\
\left.\left.\left.B u\right|_{x_{d}=0}=\varphi,\left(x^{\prime}, t\right) \in \mathbb{R}^{d-1} \times\right]-\infty, T\right]
\end{array}\right.
$$

Let $f_{1}$, resp. $\varphi_{1}$, be in $L^{2}\left(\mathbb{R}^{+} ; H_{\gamma, p}^{p-1}\left(\mathbb{R}^{d-1} \times \mathbb{R}_{t}\right)\right)$, resp. $H_{\gamma, p}^{(p-1) / 2}\left(\mathbb{R}^{d-1} \times \mathbb{R}_{t}\right)$, such that $f_{1}=f$ and $\varphi_{1}=\varphi$ for $\left.\left.t \in\right]-\infty, T\right]$ (they are extensions of $f, \varphi$ ). Let $u_{1}$ be the solution of 
the boundary value problem posed on $\mathbb{R}^{d-1} \times \mathbb{R}^{+} \times \mathbb{R}_{t}$ with forcing term $f_{1}$ and boundary data $\varphi_{1}$. The restriction of $u_{1}$ to $\left.\left.\mathbb{R}^{d-1} \times \mathbb{R}^{+} \times\right]-\infty, T\right]$ defines a solution of $(28)$ and we prove now its uniqueness. If $u_{2}$ is another solution, by linearity $u_{2}-u_{1}$ is the solution of a boundary value problem with boundary data and forcing term vanishing for $t<T$. Lemma 4 then implies $u_{2}=u_{1}$ for $t<T$.

The proof of 2) is similar. The point 3) is easily deduced from corollary 3) applied to $u_{1}$ above.

The case of the Schrödinger equation The study of the boundary value problems for the Schrödinger equation has several motivation: although in the classical physical context it is considered on the whole space, the equation may appear in fluid mechanics (e.g. as an approximation of equations for rogue waves) where the boundedness is an obvious choice, and anyway, in view of numerical analysis, it is clearly necessary to know wether given boundary conditions define a well-posed problem.

The aim of this paragraph is to prove the following result.

Theorem 5. Let $\left(u_{0}, f, \varphi\right) \in H_{0}^{1}(\Omega) \times H_{\gamma, 2}^{1}\left(\Omega \times\left[0, \infty[) \times H_{0}^{3 / 2}(\partial \Omega \times[0, \infty[)\right.\right.$. If $f$ is the limit in $H_{\gamma, 2}^{1}$ of $\left.\left.C_{c}^{\infty}(\bar{\Omega} \times] 0, T\right]\right)$ functions, then the initial boundary value problem

$$
\left\{\begin{array}{l}
\partial_{t} u+i \Delta u=f,\left(x^{\prime}, x_{d}, t\right) \in \mathbb{R}^{d-1} \times \mathbb{R}^{+} \times \mathbb{R}_{t}^{+}, \\
\left.u\right|_{x_{d}=0}=\varphi,\left(x^{\prime}, t\right) \in \mathbb{R}^{d-1} \times \mathbb{R}_{t}^{+} \\
\left.u\right|_{t=0}=u_{0}, \quad x \in \mathbb{R}^{d-1} \times \mathbb{R}^{+}
\end{array}\right.
$$

admits an unique solution $u \in \mathcal{C}\left(\mathbb{R}_{t} ; H^{1}(\Omega)\right.$ that satisfies the estimate

$$
\begin{aligned}
\forall T>0, e^{-\gamma T}\|u(T)\|_{H^{1}(\Omega)}+\|u\|_{L_{\gamma}^{2}\left([0, T] ; H^{1}\right)}+\left|\partial_{x_{d}} u(0)\right|_{H_{\gamma, 2}^{1 / 2}} & \lesssim\|f\|_{H_{\gamma, 2}^{1}(\Omega \times[0, T)} \\
& +|\varphi|_{H_{\gamma, 2}^{3 / 2}(\partial \Omega \times[0, T[)}
\end{aligned}
$$

We first wish to give a few historical hints that should enlight the ideas of proof hidden in technical arguments. Let $P\left(\partial_{t}, \partial_{x}\right)$ be a scalar differential operator, we denote by $\partial P / \partial \tau$ the operator which has for symbol $\partial P(\tau, \eta) / \partial \tau$. In 1956 L. Gårding [11] pointed out that for scalar strictly hyperbolic operators, if $P u=f$ then $(\partial P / \partial \tau) u$ is a 'good multiplier' to obtain a priori estimates for $u$. In fact, the algebraical identities initially obtained to study the strictly hyperbolic Cauchy problem proved to be sharp enough to study the IBVP too: this method was continuated by J. Rauch [21] (and Sakamoto $[23,24])$ who obtained the well-posedness of the initial boundary value problem for strictly hyperbolic first order systems and $L^{2}$ initial data.

For the Schrödinger equation, we have $\left(\partial_{\tau}\left(\tau-i|\xi|^{2}\right)=1\right.$, thus the multiplier is simply $u$. Even though it is here an obvious choice for the energy method, we believe that this method could be applied in less immediate cases. 
To begin with, we investigate the pure boundary value problem:

$$
\left\{\begin{array}{l}
\partial_{t} u+i \Delta u=f,\left(x^{\prime}, x_{d}, t\right) \in \mathbb{R}^{d-1} \times \mathbb{R}^{+} \times \mathbb{R}_{t} \\
\left.u\right|_{x_{d}=0}=\varphi,\left(x^{\prime}, t\right) \in \mathbb{R}^{d-1} \times \mathbb{R}_{t} .
\end{array}\right.
$$

Clearly, the Schrödinger operator is 2-homogeneous and strictly dispersive. Moreover, the Dirichlet boundary conditions satisfy (KLU), indeed the reformulated problem (12) reads

$$
\left\{\begin{array}{l}
\partial_{x_{d}}\left(\begin{array}{c}
\Lambda_{\gamma, 2} u \\
\partial_{x_{d}} u
\end{array}\right)=\left(\begin{array}{cc}
0 & \Lambda_{\gamma, 2} \\
\left(i \partial_{t}-\Delta^{\prime}\right) \Lambda_{\gamma, 2}^{-1} & 0
\end{array}\right)\left(\begin{array}{c}
\Lambda_{\gamma, 2} u \\
\partial_{x_{d}} u
\end{array}\right)+\left(\begin{array}{c}
0 \\
-i f
\end{array}\right) \\
\Lambda_{\gamma, 2} u=\Lambda_{\gamma, 2} \varphi
\end{array}\right.
$$

with $\Delta^{\prime}=\partial_{x_{1}}^{2}+\cdots+\partial_{x_{d-1}}^{2}$. It is easily seen that the stable subspace of $G(\tau, \eta)=$ $\left(\begin{array}{cc}0 & \lambda_{2} \\ \left(i \tau+\left|\xi^{\prime}\right|^{2}\right) / \lambda_{2} & 0\end{array}\right)$ is generated by the vector $\left(\begin{array}{c}1 \\ -\frac{\sqrt{i \tau+|\eta|^{2}}}{\lambda_{2}}\end{array}\right)$, where $\sqrt{\cdot}$ is the determination on $\mathbb{C} \backslash i \mathbb{R}^{-}$such that $\sqrt{1}=1$. For the coordinates $(\widehat{\tau}, \widehat{\eta}, \varepsilon)$ the eigenvector reads $\left(-\sqrt{i \widehat{\tau}+|\widehat{\eta}|^{2}}\right)$. According to Prop. $4,(\mathrm{KLU})$ is satisfied because $F:=\left(\begin{array}{ll}1 & 0\end{array}\right)$ is clearly an isomorphism $E^{-} \rightarrow \mathbb{C}$ for $(\widehat{\tau}, \widehat{\eta}) \in \mathbb{S}_{2}^{+}$. In particular, this implies well-posedness of the pure boundary value problem (30) for the Schrödinger equation, as well as for the dual problem associated which, as shown subsequently, is in fact the same Schrödinger equation.

Remark 9. If $d \geq 2$, due to the cancellation of the eigenvalues when $\tau=i \delta, \delta=|\eta|^{2}$, the Neumann boundary conditions do not satisfy (KLU) (at those 'glancing' points, the trace of $\partial_{x_{d}} \widehat{u}$ has to vanish, preventing the existence of a solution for arbitrary boundary data).

By the identity

$$
\begin{aligned}
\iint_{\mathbb{R}^{d-1} \times \mathbb{R}^{+} \times \mathbb{R}_{t}}\left(\partial_{t} u+i \Delta u\right) \bar{v} & =-\iint_{\mathbb{R}^{d-1} \times \mathbb{R}^{+} \times \mathbb{R}_{t}} u \overline{\left(\partial_{t} v+i \Delta v\right)} \\
& -i \int_{\mathbb{R}^{d-1} \times \mathbb{R}_{t}} \partial_{x_{d}} u(0) \overline{v(0)}+i \int_{\mathbb{R}^{d-1} \times \mathbb{R}_{t}} u(0) \overline{\partial_{x_{d}} v(0)}
\end{aligned}
$$

we see that the dual problem for the Schrödinger BVP with Dirichlet conditions is just itself. For conciseness, we will denote $\Omega:=\mathbb{R}^{d-1} \times \mathbb{R}^{+*}$.

The proof of Theorem 5 is made in four steps (of unequal difficulty):

- With Proposition 10 we prove a pointwise in time estimate for the dual BVP,

- This estimate implies an $L^{2}\left([0, T] ; H^{1}(\Omega)\right)$ estimate (42) for the solution of the IBVP (this is the most significant part), 
- The method to prove Proposition 10, enhanced with the $L^{2}\left([0, T] ; H^{1}\right)$ estimate, is applied again to obtain a pointwise in time estimate for the IBVP,

- The well-posedness is then obtained by density arguments.

Note that if $f, \varphi$ are $\mathcal{C}_{c}^{\infty}\left(\bar{\Omega} \times \mathbb{R}_{t}^{+}\right)$and vanish near $x_{d}=t=0$, there exists a smooth solution: let $\chi(t) \in C_{c}^{\infty}\left(\mathbb{R}^{+}\right)$with $\chi(0)=1, u_{c}=\chi u_{0}$. The function $\left.u_{c}\right|_{x_{d}=0}$ is smooth and compactly supported, if $u_{b}$ is the solution of the initial boundary value problem with zero initial data, boundary data $\varphi-\left.u_{c}\right|_{x_{d}=0}$ and forcing term $-\left(\partial_{t}+i \Delta\right) u_{c}+f$, then $u_{c}+u_{b}$ is a solution of $(29)$.

Proposition 10. Let $T \in \mathbb{R},\left(\varphi_{1}, f_{1}\right) \in \mathcal{C}_{c}^{\infty}\left(\partial \Omega \times\left[T, \infty[) \times \mathcal{C}_{c}^{\infty}(\bar{\Omega} \times[T, \infty[), v\right.\right.$ be the smooth solution of the (dual) boundary value problem

$$
\left\{\begin{array}{l}
\partial_{t} v+i \Delta v=f_{1},\left(x^{\prime}, x_{d}, t\right) \in \mathbb{R}^{d-1} \times \mathbb{R}^{+} \times \mathbb{R}_{t}, \\
\left.v\right|_{x_{d}=0}=\varphi_{1},\left(x^{\prime}, t\right) \in \mathbb{R}^{d-1} \times \mathbb{R}_{t}^{+}
\end{array}\right.
$$

then for $\gamma \geq \Gamma$ large enough it satisfies the pointwise a priori estimate

$$
\begin{aligned}
e^{2 \gamma T}\|v(T)\|_{L^{2}(\Omega)}^{2} & +\gamma\|v\|_{L_{-\gamma}^{2}(\Omega \times[T, \infty[)}^{2}+\sum_{j=0}^{1}\left|\partial_{x_{d}}^{j} v\right|_{H_{-\gamma, 2}^{1 / 2-j}(\partial \Omega \times[T, \infty[)}^{2} \\
& \leq C\left(\frac{1}{\gamma}\left\|f_{1}\right\|_{L_{-\gamma}^{2}(\Omega \times[T, \infty[)}^{2}+\left|\varphi_{1}\right|_{H_{-\gamma, 2}^{1 / 2}(\partial \Omega \times[T, \infty[)}^{2}\right) .
\end{aligned}
$$

Proof. Note that if we forget the term $e^{2 \gamma T}\|v(T)\|_{L^{2}(\Omega)}^{2}$ this is simply a weakened version of $(b E D s)$ (p.18) for $s=-1$, thus we only need to prove that this term is controlled by the right hand side (and possibly the other terms of the left hand side). If we multiply the equation by $\bar{v}$, integrate over $\Omega \times[T, \infty[$ and take the real part we obtain

$$
\begin{aligned}
e^{2 \gamma T} \int_{\Omega}|v(T)|^{2}+\gamma \iint_{\Omega \times[T, \infty[} e^{2 \gamma t}|v|^{2} & =\operatorname{Re}\left(-i \int_{\partial \Omega \times[T, \infty[} e^{2 \gamma t} \partial_{x_{d}} v \bar{v}\right. \\
& \left.+\iint_{\Omega \times[T, \infty[} e^{2 \gamma t} f_{1} \bar{v}\right) .
\end{aligned}
$$

To deal with the first term on the right hand side, we use the duality inequality

$$
\begin{aligned}
\left|\int_{\partial \Omega \times[T, \infty[} e^{2 \gamma t} \partial_{x_{d}} v \bar{v}\right| & \leq\left|e^{\gamma t} \partial_{x_{d}} v\right|_{H_{2}^{-1 / 2, \gamma}}\left|e^{\gamma t} v\right|_{H^{1 / 2, \gamma}} \\
& =\left|\partial_{x_{d}} v\right|_{H_{-\gamma, 2}^{-1 / 2}}|v|_{H_{-\gamma, 2}^{1 / 2}}
\end{aligned}
$$

where $H_{2}^{s, \gamma}\left(\mathbb{R}^{d-1} \times \mathbb{R}_{t}\right):=\left\{w \in L^{2}: \iint\left(|\gamma|+|\delta|+|\eta|^{2}\right)^{s}|\widehat{w}(\delta)|^{2} d \delta<\infty\right\}$ and $H_{2}^{s, \gamma}\left(\mathbb{R}^{d-1} \times\right.$ $\left[T, \infty[)\right.$ is defined by restriction. We have used here that the topological dual of $H_{2}^{1 / 2, \gamma}\left(\mathbb{R}^{d-1} \times\right.$ 
$\left[T, \infty[)\right.$ is $H_{2}^{-1 / 2, \gamma}$, which is not a trivial fact. It relies on the identification for standard Sobolev spaces $H^{s}([a, b])=H_{0}^{s}([a, b])$ when $0 \leq s<1 / 2$ (see the first chapter of the classical book of Lions and Magenes [16] for a reference on this). The term $\left\|\partial_{x_{d}} v\right\|_{H_{\gamma, 2}^{-1 / 2}}$ is estimated with $(b E D s)$ for $s=-1$ :

$$
\left|\partial_{x_{d}} v\right|_{H_{-\gamma, 2}^{-1 / 2}} \lesssim\left|\varphi_{1}\right|_{H_{-\gamma, 2}^{1 / 2}}+\left\|f_{1}\right\|_{L_{-\gamma}^{2}}
$$

The second term of the right hand side is more easily controlled, indeed a simple CauchySchwarz inequality gives

$$
\iint_{\Omega \times[T, \infty[} e^{2 \gamma t} f_{1} \bar{v} \leq\|v\|_{L_{-\gamma}^{2}}\left\|f_{1}\right\|_{L_{-\gamma}^{2}}
$$

Finally, gluing $(32,33,34)$ we obtain $(31)$.

We deduce an a priori estimate for the initial boundary value problem in negative norms. The presence of boundary terms will make the analysis in negative norms rather tedious: we define the dual norm

$$
\|u\|_{H_{\gamma}^{(-s)}}:=\sup _{\varphi \in H_{\gamma, 2}^{s}} \iint_{\bar{\Omega} \times[T, \infty[)} u \bar{\varphi}
$$

and underline that since the functions $\varphi$ above do not necessarily cancel on the boundary, this norm is not the usual norm of $H_{\gamma, 2}^{-s}$, in fact we have obviously

$$
\|\cdot\|_{H_{\gamma, 2}^{(-s)}} \leq\|\cdot\|_{H_{\gamma, 2}^{-s}}
$$

Proposition 11. Let $(\varphi, f) \in \mathcal{C}_{c}^{\infty}(\partial \Omega \times \mathbb{R}) \times \mathcal{C}_{c}^{\infty}(\Omega \times \mathbb{R})$, $u$ be the smooth solution of the IBVP

$$
\left\{\begin{array}{l}
\partial_{t} u+i \Delta u=f,\left(x^{\prime}, x_{d}, t\right) \in \mathbb{R}^{d-1} \times \mathbb{R}^{+} \times \mathbb{R}_{t}^{+}, \\
\left.u\right|_{x_{d}=0}=\varphi,\left(x^{\prime}, t\right) \in \mathbb{R}^{d-1} \times \mathbb{R}_{t}^{+}, \\
\left.u\right|_{t=0}=u_{0}, \quad x \in \mathbb{R}^{d-1} \times \mathbb{R}^{+} .
\end{array}\right.
$$

then for $\gamma \geq \Gamma$ large enough it satisfies the a priori estimate

$$
\begin{aligned}
\|u\|_{L_{\gamma, 2}^{2}(\Omega \times[0, \infty[)}^{2}+\sum_{j=0}^{1}\left|\partial_{x_{d}}^{j} u\right|_{H_{\gamma, 2}^{1 / 2-j}(\partial \Omega \times[0, \infty[)}^{2} & \lesssim\|f\|_{L_{\gamma, 2}^{2}(\Omega \times[0, \infty[)}^{2} \\
& +|\varphi|_{H_{\gamma, 2}^{1 / 2}(\partial \Omega \times[0, \infty[)}^{2}+\left\|u_{0}\right\|_{H^{(-1)}}^{2} .
\end{aligned}
$$

Remark 12. Although this estimate is not pontwise in time, it is essential because it shows that the solution of the boundary value problem with initial data satisfies an 'IBVP analogous' of $(E D)$ (p.14). Of course, a norm of the initial data had to be added in the right hand side. 
Proof. We proceed by duality. For $f_{1} \in \mathcal{C}_{c}^{\infty}(\Omega \times[0, \infty[)$, let $v$ be the solution of the dual BVP

$$
\left\{\begin{array}{l}
-\partial_{t} v-i \Delta v=f_{1},\left(x^{\prime}, x_{d}, t\right) \in \mathbb{R}^{d-1} \times \mathbb{R}^{+} \times \mathbb{R}_{t} \\
\left.v\right|_{x_{d}=0}=0,\left(x^{\prime}, t\right) \in \mathbb{R}^{d-1} \times \mathbb{R}_{t}^{+}
\end{array}\right.
$$

Then

$$
\begin{aligned}
\iint_{\Omega \times[0, \infty[} u \overline{f_{1}} & =\iint_{\Omega \times[0, \infty[} f \bar{v}-i \int_{\partial \Omega \times[0, \infty[} \varphi \overline{\partial_{x_{d}} v} \\
& +i \int_{\partial \Omega \times[0, \infty[} \partial_{x_{d}} u 0+\int_{\Omega} u_{0} \overline{\left.v\right|_{t=0}} \\
\Rightarrow\left|\iint_{\Omega \times[0, \infty[} u \overline{f_{1}}\right| & \leq\|f\|_{L_{\gamma, 2}^{2}}\|v\|_{L_{-\gamma, 2}^{2}}+|\varphi|_{H_{\gamma, 2}^{(1 / 2)}}\left|\partial_{x_{d}} v\right|_{H_{-\gamma, 2}^{-1 / 2}} \\
& +\left\|u_{0}\right\|_{L^{2}}\left\|\left.v\right|_{t=0}\right\|_{L^{2}} .
\end{aligned}
$$

Using (31) gives

$$
\left|\iint_{\Omega \times[0, \infty[} u \overline{f_{1}}\right| \lesssim\left(\|f\|_{L_{\gamma, 2}^{2}}+\left\|u_{0}\right\|_{L_{\gamma, 2}^{2}}+|\varphi|_{H_{\gamma, 2}^{1 / 2}}\right)\left\|f_{1}\right\|_{L_{-\gamma}^{2}}
$$

and taking the supremum over $f_{1} \in \mathcal{C}_{c}^{\infty}$, we obtain by density the first part of the desired inequality:

$$
\|v\|_{L_{\gamma, 2}^{2}(\Omega \times[0, \infty[)}^{2} \lesssim\|f\|_{L_{\gamma, 2}^{2}(\Omega \times[0, \infty[)}^{2}+|\varphi|_{H_{-\gamma, p}^{1 / 2}(\partial \Omega \times[0, \infty[)}^{2}+\left\|u_{0}\right\|_{L^{2}}^{2}
$$

To evaluate the boundary terms, we use the solution still denoted $v$ of the dual BVP

$$
\left\{\begin{array}{l}
-\partial_{t} v-i \Delta v=0,\left(x^{\prime}, x_{d}, t\right) \in \Omega \times \mathbb{R}_{t} \\
\left.v\right|_{x_{d}=0}=\varphi_{1},\left(x^{\prime}, t\right) \in \partial \Omega \times \mathbb{R}_{t}
\end{array}\right.
$$

with $\varphi_{1} \in \mathcal{C}_{c}^{\infty}\left(\partial \Omega \times \mathbb{R}_{t}\right)$.

The same procedure as above gives

$$
\begin{aligned}
\left|\int_{\partial \Omega \times[0, \infty[} \partial_{x_{d}} u \bar{v}\right| & \leq\left|\iint_{\Omega \times[0, \infty[} f \bar{v}\right|+\left|\int_{\partial \Omega \times[0, \infty[} \varphi \overline{\partial_{x_{d}} v}\right|+\left|\int_{\Omega} u_{0} \overline{\left.v\right|_{t=0}}\right| \\
& \lesssim\left(\|f\|_{L_{\gamma, 2}^{2}}+\left\|u_{0}\right\|_{L_{\gamma, 2}^{2}}+|\varphi|_{H_{\gamma, 2}^{1 / 2}}\right)\left\|\varphi_{1}\right\|_{H_{-\gamma, 2}^{1 / 2}}
\end{aligned}
$$

and again taking the supremum gives the second part of the desired inequality.

We are now left to raise the inequality (36) to $H^{1}$ regularity. Some simplifying hypotheses (in fact strong compatibility conditions) are made:

- the function $f$, resp. $\varphi$, is in $\mathcal{C}_{c}^{\infty}(\Omega \times] 0, \infty[)$, resp. $C_{c}^{\infty}(\partial \Omega \times] 0, \infty[)$, 
- the function $u_{0}$ is in $\mathcal{C}_{c}^{\infty}(\Omega)$.

Since $\partial_{j} u$ satisfies the Schrödinger equation

$$
\left\{\begin{array}{l}
\partial_{t} \partial_{j} u+i \Delta \partial_{j} u=\partial_{j} f,\left(x^{\prime}, x_{d}, t\right) \in \mathbb{R}^{d-1} \times \mathbb{R}^{+} \times \mathbb{R}_{t}^{+}, \\
\left.\partial_{j} u\right|_{x_{d}=0}=\left.\partial_{j} u\right|_{x_{d}=0},\left(x^{\prime}, t\right) \in \mathbb{R}^{d-1} \times \mathbb{R}_{t}^{+} \\
\left.\partial_{j} u\right|_{t=0}=\partial_{j} u_{0}, x \in \mathbb{R}^{d-1} \times \mathbb{R}^{+}
\end{array}\right.
$$

the a priori estimate (36) implies for $j \neq d$

$$
\begin{aligned}
\left\|\partial_{j} u\right\|_{L_{\gamma, 2}^{2}(\Omega \times[0, \infty[)}^{2} & +\sum_{r=0}^{1}\left|\partial_{x_{d}}^{r} \partial_{j} u\right|_{H_{\gamma, 2}^{1 / 2-j}(\partial \Omega \times[0, \infty[)}^{2} \lesssim\left\|\partial_{j} f\right\|_{L_{\gamma}^{2}(\Omega \times[0, \infty[)}^{2} \\
& +\left|\partial_{j} \varphi\right|_{H_{\gamma, 2}^{1 / 2}(\partial \Omega \times[0, \infty[)}^{2}+\left\|\partial_{j} u_{0}\right\|_{L^{2}(\Omega)}^{2} .
\end{aligned}
$$

and

$$
\begin{aligned}
\left\|\partial_{d} u\right\|_{L_{\gamma, 2}^{2}(\Omega \times[0, \infty[)}^{2} \lesssim\left\|\partial_{d} f\right\|_{L_{\gamma}^{2}(\Omega \times[0, \infty[)}^{2} & +\left|\partial_{d} u(0)\right|_{H_{\gamma, 2}^{1 / 2}(\partial \Omega \times[0, \infty[)}^{2} \\
& +\left\|\partial_{d} u_{0}\right\|_{L^{2}(\Omega)}^{2} .
\end{aligned}
$$

In particular,

$$
\begin{aligned}
\|u\|_{L_{\gamma}^{2}\left(\mathbb{R}_{t}^{+} ; H^{1}(\Omega)\right)} & \lesssim\|f\|_{L_{\gamma}^{2}\left(\mathbb{R}_{t}^{+} ; H^{1}(\Omega)\right)}+|\varphi|_{H_{\gamma, 2}^{3 / 2}}+\left\|u_{0}\right\|_{H^{1}(\Omega)}+\left|\partial_{x_{d}} u(0)\right|_{H_{\gamma, 2}^{1 / 2}}, \\
\left|\nabla^{\prime} \partial_{x_{d}} u(0)\right|_{H_{\gamma}^{-1 / 2}\left(\partial \Omega \times \mathbb{R}_{t}^{+}\right)} & \lesssim\|f\|_{L_{\gamma}^{2}\left(\mathbb{R}_{t}^{+} ; H^{1}(\Omega)\right)}+|\varphi|_{H_{\gamma, 2}^{3 / 2}}+\left\|u_{0}\right\|_{H^{1}(\Omega)} .
\end{aligned}
$$

To close the estimate, it remains to control $\left|\partial_{x_{d}} u(0)\right|_{H_{\gamma, 2}^{1 / 2}}$. We state an inequality that can be proved by following the demonstration of (31) and using simply ( $b E D)$ (p.18) instead of $(b E D s)$ :

$$
\begin{aligned}
e^{2 \gamma T}\|v(T)\|_{H^{1}(\Omega)}^{2} & +\gamma\|v\|_{H_{-\gamma, 2}^{1}(\Omega \times[T, \infty[)}^{2}+\sum_{j=0}^{1}\left|\partial_{x_{d}}^{j} v\right|_{H_{-\gamma, 2}^{3 / 2-j}(\partial \Omega \times[T, \infty[)}^{2} \\
& \lesssim\left|\varphi_{1}\right|_{H_{-\gamma, 2}^{3 / 2}(\partial \Omega \times[T, \infty[)}^{2},
\end{aligned}
$$

and derive an estimate for $\partial_{t} \partial_{d} u(0)$ by following the proof of (36). Indeed $\partial_{t} u$ is the solution of a Schrödinger equation, and if $v$ is the solution of

$$
\left\{\begin{array}{l}
-\partial_{t} v-i \Delta v=0,\left(x^{\prime}, x_{d}, t\right) \in \Omega \times \mathbb{R}_{t}, \\
\left.v\right|_{x_{d}=0}=\varphi_{1},\left(x^{\prime}, t\right) \in \partial \Omega \times \mathbb{R}_{t}
\end{array}\right.
$$

we find (the hypotheses on the support of $f, \varphi, u_{0}$ are essential here)

$$
\begin{aligned}
\left|\int_{\partial \Omega \times[0, \infty[} \partial_{t} \partial_{x_{d}} u \overline{\varphi_{1}}\right| & \leq\left|\iint_{\Omega \times[0, \infty[} f \bar{v}\right|+\left|\int_{\partial \Omega \times[0, \infty[} \partial_{t} \varphi \overline{\partial_{x_{d}} v}\right|+\left|\int_{\Omega} u_{0} \overline{\left.v\right|_{t=0}}\right| . \\
& \lesssim\left(\|f\|_{H_{\gamma, 2}^{1}}+\left\|u_{0}\right\|_{H_{\gamma, 2}^{1}}+|\varphi|_{H_{\gamma, 2}^{3 / 2}}\left\|\varphi_{1}\right\|_{H_{-\gamma, 2}^{3 / 2}}\right. \\
\Rightarrow \|\left.\partial_{t} \partial_{x_{d}} u(0)\right|_{H_{\gamma, 2}^{-3 / 2}\left(\Omega \times \mathbb{R}_{t}^{+}\right)} & \lesssim\|f\|_{H_{\gamma, 2}^{1}}+\left\|u_{0}\right\|_{H_{\gamma, 2}^{1}}+|\varphi|_{H_{\gamma, 2}^{3 / 2}}
\end{aligned}
$$


Let $\partial_{x_{d}} u(0)$ be the extension by 0 for $t<0$ of $\partial_{x_{d}} u(0)$. By the hypotheses on the supports this defines a smooth extension, thus $\partial_{t} \partial_{x_{d}} u(0)=\partial_{t} \partial_{x_{d}} u(0)$ and

$$
\left|\partial_{t} \partial_{x_{d}} u(0)\right|_{H_{\gamma, 2}^{-3 / 2}\left(\partial \Omega \times \mathbb{R}_{t}^{+}\right)}=\int_{\mathbb{R}^{d-1} \times \mathbb{R}} \frac{1}{\lambda_{2}^{3}}|\tau|^{2}\left|\widehat{\underline{\partial_{d} u(0)}}\right|^{2} d \eta d \delta
$$

The inequality above combinated with (38) imply

$$
\begin{aligned}
\int_{\mathbb{R}^{d-1} \times \mathbb{R}}\left(\frac{|\tau|^{2}}{\lambda_{2}^{3}}+\frac{|\eta|^{2}}{\lambda_{2}}\right) \underline{\mid{\widehat{\partial_{d} u(0)}}^{2}} d \eta d \delta & \lesssim\|f\|_{H_{\gamma, 2}^{1}}+\left\|u_{0}\right\|_{H_{\gamma, 2}^{1}}+|\varphi|_{H_{\gamma, 2}^{3 / 2}} \\
\Leftrightarrow \int_{\mathbb{R}^{d-1} \times \mathbb{R}}\left(|\tau|+|\delta|^{2}\right)^{1 / 2}\left|\widehat{\partial_{x_{d} u(0)}}\right|^{2} d \eta d \delta & \lesssim\|f\|_{H_{\gamma, 2}^{1}}+\left\|u_{0}\right\|_{H_{\gamma, 2}^{1}}+|\varphi|_{H_{\gamma, 2}^{3 / 2}} \\
\Leftrightarrow\left|\partial_{x_{d}} u(0)\right|_{H_{\gamma, 2}^{1 / 2}\left(\partial \Omega \times \mathbb{R}_{t}^{+}\right)} & \lesssim\|f\|_{H_{\gamma, 2}^{1}}+\left\|u_{0}\right\|_{H_{\gamma, 2}^{1}}+|\varphi|_{H_{\gamma, 2}^{3 / 2}} .
\end{aligned}
$$

The injection of this inequality in (37) finally gives

$$
\|u\|_{L_{\gamma}^{2}\left(\mathbb{R}_{t}^{+} ; H^{1}(\Omega)\right)}+\left|\partial_{x_{d}} u(0)\right|_{H_{\gamma, 2}^{1 / 2}} \lesssim\|f\|_{H_{\gamma, 2}^{1}\left(\mathbb{R}_{t}^{+} \times \Omega\right)}+|\varphi|_{H_{\gamma, 2}^{3 / 2}}+\left\|u_{0}\right\|_{H^{1}(\Omega)},
$$

To obtain the same inequality with $[0, \infty[$ replaced by $[0, T]$ for some $T>0$ it suffices to follow the proof of Corollary 3 (p.25), namely:

- prove a causality principle 'same data on $[0, T]$ implies equality of the solutions on $[0, T]^{\prime}$,

- for $(f, \varphi)$ defined on $[0, T]$, use compactly supported extensions by reflection $\left(f_{r}, \varphi_{r}\right)$ on $[0, \infty[$ (this is possible because $(f, \varphi)$ are supported in $t>0$ ) and apply (41)

$$
\begin{aligned}
\|u\|_{L_{\gamma}^{2}\left([0, T] ; H^{1}\right)}+\left|\partial_{x_{d}} u(0)\right|_{H_{\gamma, 2}^{1 / 2}} & \lesssim\left\|f_{r}\right\|_{H_{\gamma, 2}^{1}(\Omega \times[0, \infty[)}+\left|\varphi_{r}\right|_{H_{\gamma, 2}^{3 / 2}(\partial \Omega \times[0, \infty[)} \\
& \lesssim\|f\|_{H_{\gamma, 2}^{1}(\Omega \times[0, T)}+|\varphi|_{H_{\gamma, 2}^{3 / 2}(\partial \Omega \times[0, T])} .
\end{aligned}
$$

We do not detail it. The same argument applied with inequality (36) also gives

$$
\|u\|_{L_{\gamma}^{2}([0, T] \times \Omega)}+\left|\partial_{x_{d}} u(0)\right|_{H_{\gamma, 2}^{1 / 2}} \lesssim\|f\|_{L_{\gamma}^{2}(\Omega \times[0, T)}+|\varphi|_{H_{\gamma, 2}^{1 / 2}\left(\partial \Omega \times\left[0, T[)^{\circ}\right.\right.} .
$$

From the inequalities above, we deduce the key estimate with the following Proposition.

Proposition 13. Let $u$ be the smooth solution of (35). It satisfies the a priori estimate

$$
\begin{aligned}
\forall T>0, e^{-\gamma T}\|u(T)\|_{H^{1}(\Omega)}+\|u\|_{L^{2}\left([0, T] ; H^{1}\right)}+\left|\partial_{x_{d}} u(0)\right|_{H_{\gamma, 2}^{1 / 2}} & \lesssim\|f\|_{H_{\gamma, 2}^{1}(\Omega \times[0, T)} \\
& +|\varphi|_{H_{\gamma, 2}^{3 / 2}\left(\partial \Omega \times\left[0, T[)^{\circ}\right.\right.} .
\end{aligned}
$$


Proof. It is basically a repetition of the proof of (31), where we integrate on $[0, T]$ instead of $\left[T, \infty\left[\right.\right.$. We only show how to obtain an estimate for $\left\|\partial_{x_{d}} u(T)\right\|_{L^{2}(\Omega)}$ since it is the most significant point. The function $\partial_{x_{d}} u$ satisfies a Schrödinger equation, the application of (43) gives

$$
\left|\partial_{x_{d}}^{2} u\right|_{H_{\gamma, 2}^{-1 / 2}} \lesssim\left\|\partial_{x_{d}} f\right\|_{L_{\gamma}^{2}(\Omega \times[0, T]}+\left|\partial_{x_{d}} u(0)\right|_{H_{\gamma, 2}^{1 / 2}\left(\partial \Omega \times\left[0, T[)^{2}\right.\right.}
$$

Multiplying $\partial_{t} \partial_{x_{d}} u+i \Delta \partial_{x_{d}} u=\partial_{x_{d}} f$ by $e^{-2 \gamma t} \overline{\partial_{x_{d}} u}$, integrating over $\Omega \times[0, T]$ and taking the real part we obtain

$$
e^{-2 \gamma t}\left\|\partial_{x_{d}} u(T)\right\|_{L^{2}(\Omega)}^{2} \lesssim\left|\partial_{x_{d}} u(0)\right|_{H_{\gamma, 2}^{1 / 2}(\partial \Omega \times[0, T])}^{2}+\left\|\partial_{x_{d}} f_{1}\right\|_{L_{\gamma}^{2}(\Omega)}^{2}+\left\|\partial_{x_{d}} u_{0}\right\|_{L^{2}(\Omega)}^{2}
$$

Then with (42) applied to $u$ we find the expected estimate

$$
e^{-2 \gamma t}\left\|\partial_{x_{d}} u(T)\right\|_{L^{2}(\Omega)}^{2} \lesssim|\varphi|_{H_{\gamma, 2}^{3 / 2}(\partial \Omega \times[0, T])}^{2}+\left\|\partial_{x_{d}} f_{1}\right\|_{L_{\gamma}^{2}(\Omega)}^{2}+\left\|\partial_{x_{d}} u_{0}\right\|_{L^{2}(\Omega)}^{2} .
$$

We can now conclude.

Proof of Theorem 5. Let $\left(U_{n}, f_{n}, \varphi_{n}\right) \in \mathcal{C}_{c}^{\infty}(\Omega) \times \mathcal{C}_{c}^{\infty}\left(\Omega \times \mathbb{R}_{t}^{+*}\right) \times \mathcal{C}_{c}^{\infty}\left(\partial \Omega \times \mathbb{R}_{t}^{+*}\right)$ converging to $\left(u_{0}, f, \varphi\right)$ in $H_{0}^{1}(\Omega) \times H_{\gamma, 2}^{1}\left(\Omega \times\left[0, \infty[) \times H_{0}^{3 / 2}\left(\partial \Omega \times\left[0, \infty[)\right.\right.\right.\right.$. Let $u_{n}$ be the solution of the boundary value problem

$$
\left\{\begin{array}{l}
\partial_{t} u_{n}+i \Delta u_{n}=f_{n},\left(x^{\prime}, x_{d}, t\right) \in \mathbb{R}^{d-1} \times \mathbb{R}^{+} \times \mathbb{R}_{t}^{+} \\
\left.u_{n}\right|_{x_{d}=0}=\varphi_{n},\left(x^{\prime}, t\right) \in \mathbb{R}^{d-1} \times \mathbb{R}_{t}^{+} \\
\left.u_{n}\right|_{t=0}=U_{n}, x \in \mathbb{R}^{d-1} \times \mathbb{R}^{+}
\end{array}\right.
$$

By Proposition 13, $u_{n}$ is a Cauchy sequence in $C\left([0, T] ; H^{1}(\Omega)\right)$ as well as the trace $\left.\partial_{x_{d}} u_{n}\right|_{x_{d}=0}$, and we obtain the existence of a solution with the appropriate estimates. The uniqueness is proved by duality arguments as for (36). If $u$ is solution of

$$
\left\{\begin{array}{l}
\partial_{t} u+i \Delta u=0,\left(x^{\prime}, x_{d}, t\right) \in \mathbb{R}^{d-1} \times \mathbb{R}^{+} \times \mathbb{R}_{t}^{+} \\
\left.u\right|_{x_{d}=0}=0,\left(x^{\prime}, t\right) \in \mathbb{R}^{d-1} \times \mathbb{R}_{t}^{+} \\
\left.u\right|_{t=0}=0, x \in \mathbb{R}^{d-1} \times \mathbb{R}^{+}
\end{array}\right.
$$

using the dual problem

$$
\left\{\begin{array}{l}
-\partial_{t} u-i \Delta u=f_{1},\left(x^{\prime}, x_{d}, t\right) \in \mathbb{R}^{d-1} \times \mathbb{R}^{+} \times \mathbb{R}_{t}^{+}, \\
\left.u\right|_{x_{d}=0}=0,\left(x^{\prime}, t\right) \in \mathbb{R}^{d-1} \times \mathbb{R}_{t}^{+},
\end{array}\right.
$$

we find that $\int_{\Omega \times[0, \infty[} u f_{1}=0$ for any $f \in C_{c}^{\infty}(\Omega \times[0, \infty[)$, which implies $u=0$. 
Remark 14. It should be underlined (if it is not clear yet) that Theorem 5 is a very rough result. The vanishing of all data on the neighbourhood of $x_{d}=t=0$ is a too strong condition, more realistic compatibility conditions should be investigated. Moreover the regularity needed on $f$ is quite excessive, since we did not use any dispersive regularization result. In fact simply solving separately the abstract Cauchy problem

$$
\left\{\begin{array}{l}
\partial_{t} u+i \Delta u=f,\left(x^{\prime}, x_{d}, t\right) \in \mathbb{R}^{d-1} \times \mathbb{R}^{+} \times \mathbb{R}_{t}^{+}, \\
\left.u\right|_{x_{d}=0}=0,\left(x^{\prime}, t\right) \in \mathbb{R}^{d-1} \times \mathbb{R}_{t}^{+}, \\
\left.u\right|_{t=0}=0, x \in \mathbb{R}^{d-1} \times \mathbb{R}^{+} .
\end{array}\right.
$$

with semi groups arguments would very likely have allowed to suppress the time regularity of $f$. We did not go this way for conciseness reasons, and also because this would have lead to loss of regularity for $\left.\partial_{x_{d}} u\right|_{x_{d}=0}$.

Conclusion: The method of Kreiss symmetrizers has allowed us to derive a priori estimates for a wide class of pure boundary value problems under a natural generalized Kreiss-Lopatinskiı condition. From these estimates we have deduced well-posedness results for the pure boundary value problem, and the boundary value problem with zero initial data. The regularity of the trace is higher than the one expected simply from trace theorems, this is certainly an unavoidable dispersive effect (see the results on KdV $[2,9]$ for example) although it is not clear yet wether we worked here in 'optimal' spaces. The analysis for the Schrödinger equations poses several 'immediate' problems:

- can this analysis be repeated for any strictly dispersive equations?

- what are the 'good' compatibility conditions?

We underline moreover that the derivation of a priori estimates relies quite heavily on the fact that we work with constant coefficients on a half space. The investigation of more realistic non-homogeneous boundary value problems (variable coefficients for the leading terms as well as curved boundary) remains a difficult question.

\section{A Construction of Generalized Kreiss symmetrizers}

We sketch here the proof of theorem 2. It consists in constructing local Kreiss symmetrizer, i.e. a symbol defined on the neighbourhood of points $(\widehat{\tau}, \widehat{\eta}, \varepsilon)$ satisfying $(K 1, K 2)$ (p.13) reformulated for $(\widehat{\tau}, \widehat{\eta}, \varepsilon)$ variables, and then use a partition of unity to obtain a symbol defined on $\mathbb{S}_{p}^{+} \times\left[0, \varepsilon_{0}\right]$ for $\varepsilon_{0}$ small enough. The diffeomorphism (16) then gives a symbol defined on $\mathcal{E}^{1 / \varepsilon_{0}^{p}}$ which is the symbol of a generalized Kreiss symmetrizer.

We first perform the easy but significant construction for non glancing points, which explains why the natural minorant is $c(\tau, \eta)=\alpha / \lambda_{p}^{p-1}$. The notations are those of section 
1: $g(\tau, \eta)$ is a smooth symbol, such that $\operatorname{det}\left(g-i \omega I_{n m p}\right)$ is a polynomial in $(\tau, \eta, \zeta)$ whose roots in $\tau$ are purely imaginary and simple for $(\eta, \omega) \in \mathbb{R}^{d} \backslash\{0\}$. It is more convenient to work with the variables

$$
(\widehat{\tau}, \widehat{\eta}, \varepsilon):=\left(\frac{\tau}{\lambda_{p}^{p}}, \frac{\eta}{\lambda_{p}}, \frac{1}{\lambda_{p}}\right),
$$

and the bounded matrice $h((\widehat{\tau}, \widehat{\eta}, \varepsilon))=\varepsilon g((\widehat{\tau}, \widehat{\eta}, \varepsilon))$, which is smooth for $(\widehat{\tau}, \widehat{\eta}, \varepsilon) \in$ $\mathbb{S}_{p}^{+} \times[0,1]$. Since $\widehat{\tau}=\tau / \lambda_{p}^{p}$, the hypotheses $\left.(K 1), K 2\right)$ p.13 can be rewritten for $c(\tau, \eta)=$ $\alpha / \lambda_{p}^{p-1}$ as

$$
\operatorname{Re}(s h) \geq \alpha \operatorname{Re}(\widehat{\tau}), s(\widehat{\tau}, \widehat{\eta}, \varepsilon) \geq I-C F^{*} F(\widehat{\tau}, \widehat{\eta}, \varepsilon) .
$$

We denote $\chi(\zeta ;(\widehat{\tau}, \widehat{\eta}, \varepsilon)):=\chi_{h(\widehat{\tau}, \widehat{\eta}, \varepsilon)}(\zeta)=\operatorname{det}(h((\widehat{\tau}, \widehat{\eta}, \varepsilon))-\zeta I)$ the characteristic polynomial of $h$. Note that it is a polynomial in $(\widehat{\tau}, \widehat{\eta}, \varepsilon)$.

The case of non-glancing points: the point $\left(\widehat{\tau}_{0}, \widehat{\eta}_{0}, 0\right)$ is said to be non-glancing if the purely imaginary roots in $\zeta$ of $\chi\left(\zeta ; \widehat{\tau_{0}}, \widehat{\eta_{0}}, 0\right)$ are simple. If there are purely imaginary eigenvalues Proposition 1 implies $\operatorname{Re}(\widehat{\tau})=0$, thus we may assume without loss of generality that $(\widehat{\tau}, \widehat{\eta}, \varepsilon)=(i \widehat{\delta}, \widehat{\eta}, 0)$. If the purely imaginary eigenvalues are simple, by continuity they remain simple on a neighbourhood of $\left(\widehat{\tau_{0}}, \widehat{\eta}_{0}, 0\right)$. Thus there is a smooth change of basis such that on a neighbourhood of $\left(\widehat{\tau_{0}}, \widehat{\eta_{0}}, 0\right)$ the matrix $h((\widehat{\tau}, \widehat{\eta}, \varepsilon))$ has the form

$$
h^{\prime}:=P^{-1} h P=\left(\begin{array}{cccccc}
\lambda_{1} & 0 & & & & \\
0 & \lambda_{2} & & & & \\
& & \ddots & & & \\
& & & \lambda_{r} & & \\
& & & & e_{-} & \\
& & & & & e_{+}
\end{array}\right)
$$

with $e_{ \pm}$square matrices of size $\mu_{ \pm}$, spectrum $S_{p}\left(e_{ \pm}\right) \subset\{ \pm \operatorname{Re}(z)>0\}, \lambda\left(\widehat{\tau_{0}}, \widehat{\eta_{0}}, \varepsilon_{0}\right)=$ $i \omega_{j} \in i \mathbb{R}$. Let $r_{-}$, resp $r_{+}$, be the number of $\lambda_{j}$ such that

$$
\operatorname{Re}\left(\lambda _ { j } ( ( \widehat { \tau } , \widehat { \eta } , \varepsilon ) ) > 0 \text { if } \operatorname { R e } ( \widehat { \tau } ) > 0 , \text { resp. } \operatorname { R e } \left(\lambda_{j}((\widehat{\tau}, \widehat{\eta}, \varepsilon))<0 \text { if } \operatorname{Re}(\widehat{\tau})>0 .\right.\right.
$$

Up to a reindexation, $h^{\prime}$ reads

$$
h^{\prime}=\left(\begin{array}{cccccccc}
\lambda_{1}^{-} & 0 & & & & & & \\
0 & \ddots & & & & & & \\
& & \lambda_{r_{-}}^{-} & & & & & \\
& & & \lambda_{1}^{+} & & & & \\
& & & & \ddots & & & \\
& & & & & \lambda_{r_{+}}^{+} & & \\
& & & & & & e_{-} & \\
& & & & & & & e_{+}
\end{array}\right)
$$


For the construction of symmetrizers, the blocks $e_{ \pm}$are easily handled, and the natural idea for the purely imaginary eigenvalue is to give them the 'good sign'. The next proposition quantifies this.

Proposition 15. The exists a neighbourhood of $\left(\widehat{\tau}_{0}, \widehat{\eta}_{0}, 0\right)$, positive hermitian matrices $s_{ \pm}$and $\kappa>0$ large enough such that

$$
s:=\left(\begin{array}{cccc}
-I_{r_{-}} & & & \\
& \kappa I_{r_{+}} & & \\
& & -s_{-} & \\
& & & \kappa s_{+}
\end{array}\right)
$$

defines a local symmetrizer for $h^{\prime}$ with $c(\widehat{\tau}, \widehat{\eta}, \varepsilon)=\varepsilon^{p-1} \alpha$.

Proof. The existence of $s_{ \pm}$positive hermitians such that $\operatorname{Re}\left(s_{ \pm} e_{ \pm}\right) \geq I$ is classical, see for example lemma 9.2 p.238.

The simplicity of the purely imaginary eigenvalues and the hypothesis $(D s)$ imply

$$
\partial_{\widehat{\tau}} \chi\left(i \omega_{j}^{ \pm},\left(\widehat{\tau_{0}}, \widehat{\eta_{0}}, 0\right)\right) \neq 0, \partial_{\zeta} \chi\left(i \omega_{j}^{ \pm},\left(\widehat{\tau_{0}}, \widehat{\eta_{0}}, 0\right)\right) \neq 0
$$

We deduce from the implicit functions theorem that

$$
\partial_{\widehat{\tau}} \lambda_{j}^{ \pm}\left(\left(\widehat{\tau_{0}}, \widehat{\eta}_{0}, 0\right)\right) \in \mathbb{R}^{*} \text { and thus } \pm \operatorname{Re}\left(\lambda_{j}^{ \pm}((\widehat{\tau}, \widehat{\eta}, \varepsilon))\right) \geq \alpha \operatorname{Re}(\widehat{\tau}),
$$

for some $\alpha>0$. This gives then for $\kappa \geq 1$

$$
\begin{aligned}
\operatorname{Re}\left(s h^{\prime}\right)= & \left(\begin{array}{ccccc}
-\operatorname{Re}\left(\lambda_{1}^{-}\right) & 0 & & & \\
0 & \ddots & & & \\
& & \operatorname{Re}\left(\lambda_{1}^{+}\right) & & \\
& & & \ddots & \\
& & & \operatorname{Re}\left(s_{-} e_{-}\right) & \\
& & & & \operatorname{Re}\left(s_{+} e_{+}\right)
\end{array}\right) \\
& \geq\left(\begin{array}{ccc}
\alpha \operatorname{Re}(\widehat{\tau}) I_{r_{-}+r_{+}} & & \\
& I_{\mu_{-}} & \\
& & I_{\mu_{+}}
\end{array}\right) \geq \min (\alpha, 1) \operatorname{Re}(\widehat{\tau}),
\end{aligned}
$$

which is exactly $(K 1)$.

The verification of $(K 2)$ is based on more algebraical arguments since by continuity it is sufficient to check it at the point $\left(\widehat{\tau}_{0}, \widehat{\eta}_{0}, 0\right)$. First of all, it is clear that the vectors of the extended stable subspace $E^{-}\left(\left(\widehat{\tau_{0}}, \widehat{\eta}_{0}, 0\right)\right)$ are precisely of the form $\left(x_{1}, \cdots, x_{r_{-}}, 0, \cdots, 0, y_{1}, \cdots, y_{\mu_{-}}, 0, \cdots, 0\right)$. If $\Pi_{-}$is the projection on $E^{-}$, we write $X=\Pi_{-} X+\left(I-\Pi_{-} X\right):=X_{-}+X_{+}$. Then

$$
s X \cdot X \geq \kappa\left|X_{+}\right|^{2}-\max \left(1,\left\|e_{-}\right\|\right)\left|X_{-}\right|^{2}
$$


We now use the inequality

$$
|X|^{2} \leq C\left(|F X|^{2}+\left|X_{+}\right|^{2}\right)
$$

which is a consequence of (KLU): indeed if it is false we could find $X_{n}$ with $\left|X_{n}\right|=1$ and $\left|F X_{n}\right|+\left|\left(X_{n}\right)_{+}\right| \rightarrow 0$. Up to extracting a subsequence it has a limit $X_{\infty}$ such that $\left|X_{\infty}\right|=1, \quad\left(X_{\infty}\right)_{+}=0$ and $F X_{\infty}=0$. This implies $X_{\infty}=\Pi_{-} X_{\infty}$ and from (KLU) we have $X_{\infty}=0$, this is a contradiction.

Injecting (50) in (49) we get

$$
s X \cdot X \geq \kappa|X|^{2}-C \kappa|F X|^{2}-\max \left(1,\left\|e_{-}\right\|\right)\left|X_{-}\right|^{2} \geq|X|^{2}-C|F X|^{2},
$$

provided $\kappa \geq 1+\max \left(1,\left\|e_{-}\right\|\right)$.

The general case: The construction for the neighbourhood of glancing points occur is notably harder than in the previous paragraph. In fact it relies heavily on the fact that the roots in $\tau$ of $\chi(i \omega, \widehat{\tau}, \widehat{\eta}, \varepsilon)$ are simple, and thus does not work for any $\varepsilon$. Nevertheless, the simplicity assumption is always true when $\varepsilon=0$, and it may be proved by following the method of Kreiss [15] (see also [6]) that on the neighbourhood of $\left(\widehat{\tau_{0}}, \widehat{\eta}_{0}, 0\right)$ the matrice has a particular block structure: if $\mathcal{V}\left(\widehat{\tau_{0}}, \widehat{\eta_{0}}, 0\right)$ is a neighbourhood of $\left(\widehat{\tau_{0}}, \widehat{\eta_{0}}, 0\right)$, then

$$
\begin{aligned}
\exists P \in \mathcal{C}^{\infty}\left(\mathcal{V}\left(\widehat{\tau_{0}}, \widehat{\eta_{0}}, 0\right)\right): \forall(\widehat{\tau}, \widehat{\eta}, \varepsilon) \in \mathcal{V}\left(\widehat{\tau_{0}}, \widehat{\eta_{0}}, 0\right), \\
P^{-1} h P(\widehat{\tau}, \widehat{\eta}, \varepsilon)=\left(\begin{array}{llll}
e_{+} & & & \\
& e_{-} & & \\
& & e_{1} & \\
& & & \ddots
\end{array}\right)
\end{aligned}
$$

with $\pm S_{p}\left(e_{ \pm}\right) \subset\{\operatorname{Re}(z)>\}$ and the $e_{j}$ 's satisfy

$$
e_{j}\left(\widehat{\tau_{0}}, \widehat{\eta_{0}}, 0\right)=\left(\begin{array}{cccc}
i \omega_{j} & i & & \\
& \ddots & \ddots & \\
& & i \omega_{j} & i \\
& & & i \omega_{j}
\end{array}\right)
$$

moreover the lower left hand coefficient of $\partial_{\operatorname{Re}(\widehat{\tau})} e_{j}$ is in $\mathbb{R}^{*}$.

In the hyperbolic frame this is the so called 'block structure condition', introduced by Majda [17] who proved that the existence of such a structure is sufficient to perform the local construction of Kreiss symmetrizers.

The reader may find in [15] or [6] a full proof of the existence of local symmetrizer in the hyperbolic frame. For the reader already used to Kreiss symmetrizers, we emphasize 
here a noticeable difference: in the hyperbolic settings, the method of Kreiss consists in building symmetrizers which are homogeneous functions of $(\tau, \eta)$, and in fact the construction is only made on the half sphere $\mathbb{S}_{p}^{+}$(this is underlined by Metivier in [19] paragraph 2.3 or Benzoni and Serre [1] 9.1.3). Because $c(\tau, \eta) \rightarrow 0$ when $\lambda_{p} \rightarrow \infty$, one may check that it is not possible to only consider the $p$-principal symbol and neglect lower order terms (the example of $\partial_{t}+c \partial_{x} u+i \partial_{x}^{2} u=0$ for $c \in \mathbb{C}$ is quite enlightening) and thus the symmetrizer can not be a $p$-homogeneous function. Fortunately the method of Kreiss is flexible enough to construct local symbols on the neighbourhood in $\mathbb{S}_{p}^{+} \times[0,1]$ of any point $\left(\widehat{\tau_{0}}, \widehat{\eta}_{0}, 0\right)$ (this is of course stronger than the construction on a neighbourhood in $\left.\mathbb{S}_{p}^{+} \times\{0\}\right)$. Note that it is indeed what we have done for the simpler case of non glancing points in the previous paragraph. By compacity of $\mathbb{S}_{p}^{+}$, there exists a finite family of neighbourhoods $\mathcal{V}_{j}:=\mathcal{V}\left(\widehat{\tau_{j}}, \widehat{\eta}_{j}, 0\right)$ such that the projection on $\mathbb{S}_{p}^{+}$of the $\mathcal{V}_{j}$ 's covers $\mathbb{S}_{p}^{+}$, it is then easy to check that $\cup \mathcal{V}_{j}$ contains $\mathbb{S}_{p}^{+} \times\left[0, \varepsilon_{0}\right]$ for some $\varepsilon_{0}>0$ small enough.

Let $s_{j}$ be the local symmetrizers corresponding to $\mathcal{V}_{j}$, each of them satisfies (on its domain of definition)

$$
\begin{gathered}
\operatorname{Re}\left(s_{j} g(\widehat{\tau}, \widehat{\eta}, \varepsilon)\right) \geq \frac{\alpha_{j}}{\varepsilon} \operatorname{Re}(\widehat{\tau}), \\
s_{j}(\widehat{\tau}, \widehat{\eta}, \varepsilon) \geq I-C_{j} F^{*} F .
\end{gathered}
$$

With a partition of unity $\chi_{j}$ we obtain a global symbol $s=\sum \chi_{j} s_{j}$ defined on $\mathbb{S}_{p}^{+} \times\left[0, \varepsilon_{0}\right]$ which satisfies $(K 1, K 2)$ :

$$
\begin{array}{r}
\operatorname{Re}(s g)=\sum \operatorname{Re}\left(\chi_{j} s_{j} g\right) \geq \frac{\min \left(\alpha_{j}\right)}{\varepsilon} \operatorname{Re}(\widehat{\tau}) \\
s=\sum \chi_{j} s_{j} \geq I-\max \left(C_{j}\right) F^{*} F .
\end{array}
$$

The diffeomorphism between $\left.\left.\mathbb{S}_{p}^{+} \times\right] 0, \varepsilon_{0}\right]$ and $\left\{(\tau, \eta) \mathcal{E}^{+}: \lambda_{p}(\tau, \eta) \geq 1 / \varepsilon_{0}\right\}$ then gives a symmetrizer defined on $\mathcal{E}^{\Gamma}$ for $\Gamma=1 / \varepsilon_{0}^{p}$.

The case of operators with 'elliptic spatial part': We consider here operators such that the determinant of their symbol $\sigma_{P}(\tau, \xi)$ satisfies $\sigma_{P}(0, \xi) \neq 0$ for any $\xi \in$ $\mathbb{R}^{d} \backslash\{0\}$. Our aim is here to prove that for such an operator, the symmetrizer satisfies $s g \geq \alpha /|\tau|^{1-1 / p}$ for some $\alpha>0$. The proof relies on two facts

- for $\delta$ small enough and $|\xi|=1, \operatorname{princ}\left(\sigma_{P}(i \delta, \xi) \neq 0\right.$,

- if the characteristic polynomial of $h \chi(\zeta ;(\widehat{\tau}, \widehat{\eta}, \varepsilon))$ has no purely imaginary roots in $\zeta$, then the symmetrizer constructed on the neighbourhood of $(\widehat{\tau}, \widehat{\eta}, \varepsilon)$ satisfies $s h \geq c$ with $c$ a positive constant only depending on the neighbourhood. 
The first point is a direct consequence of the definition and the compacity of $\{\xi:|\xi|=1\}$, the second point may be deduced from the construction performed for non glancing points. Indeed, since there are no purely imaginary $\operatorname{roots}((\widehat{\tau}, \widehat{\eta}, \varepsilon)$ is said to be an elliptic point) after a change of basis we can reduce $h$ to the simpler form $\left(\begin{array}{ll}e_{-} & \\ & e_{+}\end{array}\right)$ and we have $\operatorname{Re}\left(s h^{\prime}\right)=\left(\begin{array}{cc}s_{-} e_{-} & \\ & s_{+} e_{+}\end{array}\right) \gtrsim 1$. From the first point we deduce that there exists $\beta>0$ such that for $\widehat{\delta_{0}}<\beta$, the point $\left(\widehat{\delta_{0}}, \widehat{\eta}_{0}, 0\right)$ is an elliptic point. We recall too that, by Lemma 1 , any $\left(\widehat{\tau_{0}}, \widehat{\eta_{0}}, 0\right)$ with $\operatorname{Re}\left(\widehat{\tau_{0}}\right)>0$ is an elliptic point.

Thus on the set $\left\{(\widehat{\tau}, \widehat{\eta}, 0) \in \mathbb{S}_{p}^{+} \times\{0\}:|\operatorname{Im}(\widehat{\tau})|<\beta\right\}$, the local symmetrizers satisfy $s h \geq c$, while on its complement we have the same estimate as before $s h \geq \alpha \operatorname{Re}(\widehat{\tau})$. This rewrites for the original variables for $(\tau, \eta) \in \mathcal{E}^{\Gamma}, \Gamma$ large enough

$$
\begin{array}{cl}
\text { if } \quad & |\delta|<\beta \lambda_{p}^{p} \Rightarrow s g \geq c \lambda_{p} \geq c(\operatorname{Re}(\tau))^{1 / p} \geq c \operatorname{Re}(\tau) /|\tau|^{(p-1) / p}, \\
\text { else } & s g \geq \alpha / \lambda_{p}^{p-1},
\end{array}
$$

the condition $\delta>\beta \lambda_{p}^{p}$ implies $|\delta| \gtrsim|\eta|^{p}$, which means for $s g$

$$
s g \geq \alpha \operatorname{Re}(\tau) /\left(|\tau|^{2}+|\eta|^{2 p}\right)^{(p-1) /(2 p)} \gtrsim \alpha \operatorname{Re}(\tau) /|\tau|^{1-1 / p} .
$$

Finally, up to decreasing $\alpha$ we have, for any $(\tau, \eta) \in \mathcal{E}^{\Gamma}, \operatorname{Re}(s g) \geq \alpha \operatorname{Re}(\tau) /|\tau|^{1-1 / p}$, this completes the proof.

\section{Acknowledgements}

The author thanks Sylvie Benzoni-Gavage for guidance in this work and her help during the redaction of the article.

\section{References}

[1] Sylvie Benzoni-Gavage and Denis Serre. Multidimensional hyperbolic partial differential equations. Oxford Mathematical Monographs. The Clarendon Press Oxford University Press, Oxford, 2007. First-order systems and applications.

[2] J. Bona, M. Sun, and B. Zhang. A non homogeneous boundary value problem for the korteweg de vries equation in a quarter plane. Trans. AMS, 354:427-490, 2002.

[3] Jerry L. Bona, Shu Ming Sun, and Bing-Yu Zhang. A nonhomogeneous boundaryvalue problem for the Korteweg-de Vries equation posed on a finite domain. Comm. Partial Differential Equations, 28(7-8):1391-1436, 2003. 
[4] N. Burq. Smoothing effect for Schrödinger boundary value problems. Duke Math. J., 123(2):403-427, 2004.

[5] N. Burq, P. Gérard, and N. Tzvetkov. On nonlinear Schrödinger equations in exterior domains. Ann. Inst. H. Poincaré Anal. Non Linéaire, 21(3):295-318, 2004.

[6] Jacques Chazarain and Alain Piriou. Introduction à la théorie des équations aux dérivées partielles linéaires. Gauthier-Villars, Paris, 1981.

[7] Jean-François Coulombel. Weak stability of nonuniformly stable multidimensional shocks. SIAM J. Math. Anal., 34(1):142-172 (electronic), 2002.

[8] A. V. Faminskii. An initial boundary-value problem in a half-strip for the Kortewegde Vries equation in fractional-order Sobolev spaces. Comm. Partial Differential Equations, 29(11-12):1653-1695, 2004.

[9] Andrei V. Faminskii and Nikolai A. Larkin. Initial-boundary value problems for quasilinear dispersive equations posed on a bounded interval. Electron. J. Differential Equations, pages No. 01, 20, 2010.

[10] A.S. Fokas and L.Y. Sung. Initial boundary value problems for linear dispersive evolution equations on the half-line. Industrial mathematics institute at the University of South Carolina Technical report, 1999.

[11] Lars Gärding. Solution directe du problème de Cauchy pour les équations hyperboliques. In La théorie des équations aux dérivées partielles. Nancy, 9-15 avril 1956, Colloques Internationaux du Centre National de la Recherche Scientifique, LXXI, pages 71-90. Centre National de la Recherche Scientifique, Paris, 1956.

[12] S. G. Gindikin and L. R. Volevich. Mixed problem for partial differential equations with quasihomogeneous principal part, volume 147 of Translations of Mathematical Monographs. American Mathematical Society, Providence, RI, 1996. Translated from the Russian manuscript by V. M. Volosov.

[13] Oana Ivanovici. Precised smoothing effect in the exterior of balls. Asymptot. Anal., 53(4):189-208, 2007.

[14] Carlos E. Kenig, Gustavo Ponce, and Luis Vega. Well-posedness of the initial value problem for the Korteweg-de Vries equation. J. Amer. Math. Soc., 4(2):323-347, 1991.

[15] Heinz-Otto Kreiss. Initial boundary value problems for hyperbolic systems. Comm. Pure Appl. Math., 23:277-298, 1970. 
[16] J.-L. Lions and E. Magenes. Problèmes aux limites non homogènes et applications. Vol. 1. Travaux et Recherches Mathématiques, No. 17. Dunod, Paris, 1968.

[17] A. Majda. The stability of multidimensional shock fronts. Mem. Amer. Math. Soc., 41(275):iv+95, 1983.

[18] Guy Métivier. The block structure condition for symmetric hyperbolic systems. Bull. London Math. Soc., 32(6):689-702, 2000.

[19] Guy Métivier. Stability of multidimensional shocks. In Advances in the theory of shock waves, volume 47 of Progr. Nonlinear Differential Equations Appl., pages 25-103. Birkhäuser Boston, Boston, MA, 2001.

[20] Fabrice Planchon and Luis Vega. Bilinear virial identities and applications. Ann. Sci. Éc. Norm. Supér. (4), 42(2):261-290, 2009.

[21] Jeffrey Rauch. $\mathcal{L}_{2}$ is a continuable initial condition for Kreiss' mixed problems. Comm. Pure Appl. Math., 25:265-285, 1972.

[22] Monique Sablé-Tougeron. Existence pour un problème de l'élastodynamique Neumann non linéaire en dimension 2. Arch. Rational Mech. Anal., 101(3):261-292, 1988.

[23] Reiko Sakamoto. Mixed problems for hyperbolic equations. I. Energy inequalities. J. Math. Kyoto Univ., 10:349-373, 1970.

[24] Reiko Sakamoto. Mixed problems for hyperbolic equations. II. Existence theorems with zero initial datas and energy inequalities with initial datas. J. Math. Kyoto Univ., 10:403-417, 1970.

[25] L. R. Volevič and S. G. Gindikin. Energy estimates in a mixed problem for $(2 b+1)$ hyperbolic equations. Akad. Nauk SSSR Inst. Prikl. Mat. Preprint, (137):63, 1978. 